\title{
Contour dynamics of incompressible 3-D fluids in a porous medium with different densities
}

\author{
Diego Córdoba and Francisco Gancedo
}

\begin{abstract}
We consider the problem of the evolution of the interface given by two incompressible fluids through a porous medium, which is known as the Muskat problem and in two dimensions it is mathematically analogous to the two-phase Hele-Shaw cell. We focus on a fluid interface given by a jump of densities, being the equation of the evolution obtained using Darcy's law. We prove local well-posedness when the smaller density is above (stable case) and in the unstable case we show ill-posedness.
\end{abstract}

\section{Introduction}

The evolution of a fluid in a porous medium is an important and interesting topic of fluid mechanics (see [3]). This phenomena is based on an experimental physical principle given by H. Darcy in 1856 . Darcy's law for a 3-D fluid is given by the momentum equation

$$
\frac{\mu}{\kappa} v=-\nabla p-(0,0, \mathrm{~g} \rho)
$$

where $v$ is the incompressible velocity, $p$ is the pressure, $\mu$ is the dynamic viscosity, $\kappa$ is the permeability of the medium, $\rho$ is the liquid density and $\mathrm{g}$ is the acceleration due to gravity.

A different problem is the motion of a 2-D fluid in a Hele-Shaw cell (see [12]). In this case the fluid is set between two fixed parallel plates. These plates are close enough in such a way that the mean velocity is described by

$$
\frac{12 \mu}{b^{2}} v=-\nabla p-(0, \mathrm{~g} \rho)
$$

where $b$ denotes the distance between the plates.

Considering that the fluid in the porous medium only moves in two directions suppressing one of the variables in the horizontal plane, these two different physical phenomena of fluid dynamics become nevertheless mathematically analogous if we identify the permeability of the medium $\kappa$ and the constant $b^{2} / 12$.

The Muskat problem (see [14]) and the two-phase Hele-Shaw flow (see [17]) model the evolution of an interface between two fluids (in a porous medium and in a Hele-Shaw cell respectively) with different viscosities and densities. A lot of information can be found in the literature about both problems (see references in [9] and [13]). These free boundary problems are considered with surface tension using the Laplace-Young condition and also without surface tension in which case the pressures are equal on the interface. With surface tension, in the two dimensional case has been proven that the problems have classical solutions (see [11]). Without surface tension, Siegel, Caflisch and Howison [18] proved ill-posedness in an unstable 2-D case, namely when the higher-viscosity fluid contracts, and they show global-in-time existence of small initial data in the stable case when the higher-viscosity fluid expands. The results rely on the assumption that the Atwood number

$$
A_{\mu}=\frac{\mu_{1}-\mu_{2}}{\mu_{1}+\mu_{2}}
$$




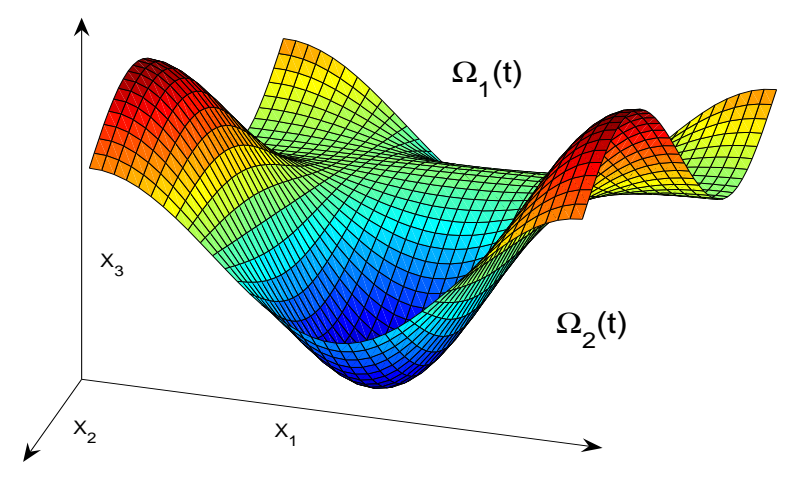

is nonzero where $\mu_{1}$ and $\mu_{2}$ are the viscosities of the fluids. In the same year, Ambrose [1] treated the 2 -D problem with an initial data fulfilling

$$
\left(\rho_{2}-\rho_{1}\right) \mathrm{g} \cos (\theta(\alpha, 0))+2 A_{\mu} U(\alpha, 0)>0,
$$

and the following condition

$$
\frac{\left(x(\alpha, 0)-x\left(\alpha^{\prime}, 0\right)\right)^{2}+\left(y(\alpha, 0)-y\left(\alpha^{\prime}, 0\right)\right)^{2}}{\left(\alpha-\alpha^{\prime}\right)^{2}}>0,
$$

where the interface is the curve $(x(\alpha, t), y(\alpha, t)), \rho_{1}$ and $\rho_{2}$ are the densities of the fluids, $\theta$ is the angle that the tangent to the curve forms with the horizontal and $U$ is the normal velocity (given by the Birkhoff-Rott integral).

We are interested in the case $A_{\mu}=0$ that presents the evolution of the interface for different densities. This case, for example, models moist and dry regions in a porous medium. Meanwhile the work of Ambrose is based on the arclength and the tangent angle formulation used by Hou, Lowengrub and Shelley [13], due to the particular form of the vorticity in the case $A_{\mu}=0$, we get to parameterize the curve in the two dimensional problem getting the condition (1) for any time (see equation (16)).

The free boundary problems given by fluids with different densities are been intensely studied. Notice the classical paper of Taylor [22] and the works of Wu [23] and [24] where the full water wave problem is solved considering the water with positive density and the air with zero density. A study of the two-dimensional case can be found in [2] due to Ambrose and Masmoudi.

In order to simplify the notation, we consider $\mu / k=12 \mu / b^{2}=1$ and $\mathrm{g}=1$. Thus, the 3 -D system is written as

$$
v\left(x_{1}, x_{2}, x_{3}, t\right)=-\nabla p\left(x_{1}, x_{2}, x_{3}, t\right)-\left(0,0, \rho\left(x_{1}, x_{2}, x_{3}, t\right)\right),
$$

where $\left(x_{1}, x_{2}, x_{3}\right) \in \mathbb{R}^{3}$ are the spatial variables and $t \geq 0$ denotes the time. Here $\rho$ is defined by

$$
\rho\left(x_{1}, x_{2}, x_{3}, t\right)=\left\{\begin{array}{lll}
\rho_{1} & \text { in } & \Omega_{1}(t) \\
\rho_{2} & \text { in } & \Omega_{2}(t),
\end{array}\right.
$$

with $\rho_{1}, \rho_{2} \geq 0$ constants and $\rho_{1} \neq \rho_{2}$.

We show in section 2 that in this case it is not necessary to assume any condition on the pressure along the interface to obtain the contour equation. Furthermore, we illustrate below that the solutions to this model are weak solutions to the following conservation of mass equation

$$
\frac{D \rho}{D t}=\rho_{t}+v \cdot \nabla \rho=0
$$


where $\operatorname{div} v=0$.

We notice the similarity with the 2-D vortex patch problem given by the two-dimensional Euler equation where the vorticity is conserved along trajectories in a weak sense. The vorticity is considered to be a characteristic function of a domain. Chemin [8] proved global-in-time existence using paradifferential calculus. A simpler proof can be found in [4] due to Bertozzi and Constantin.

In section 2 we show that due to (2) the velocity can be determined from the density by singular integral operators (see [21]). It makes the equation more singular than the 2 -D vortex patch problem where the velocity is given by the Biot-Savart law.

A singular problem, more analogous to (3), is the evolution of the 2-D quasi-geostrophic (QG) equation for sharp fronts. The QG equation models the dynamics of cold and hot air and the formation of fronts. Here the temperature $\theta$ is conserved along particle trajectories and the velocity is given by singular integral operators in the following form

$$
v=\left(-R_{2} \theta, R_{1} \theta\right),
$$

where $R_{1}$ and $R_{2}$ are the Riesz transforms (see [10] for more details of the QG equation). Rodrigo [19] proposed the contour equation of the sharp fronts where the temperature is concentrated in a domain and proved local existence and uniqueness.

The paper is organized as follows. In section 2 we derive the contour equation. We show that this equation fulfills the conservation of mass equation in section 3 . In section 4 we prove local existence and uniqueness of the stable case. In section 5 we get a family of global solutions of the 2-D stable case with small initial data. Finally, as a consequence of the previous section, in 6 we prove ill-posedness for the 3-D unstable case.

\section{The Contour Equation}

We consider the equation with $\left(x_{1}, x_{2}, x_{3}\right) \in \mathbb{R}^{3}$ where the fluid has different densities, that is $\rho$ is represented by

$$
\rho\left(x_{1}, x_{2}, x_{3}, t\right)= \begin{cases}\rho_{1}, & \left\{x_{3}>f\left(x_{1}, x_{2}, t\right)\right\} \\ \rho_{2}, & \left\{x_{3}<f\left(x_{1}, x_{2}, t\right)\right\}\end{cases}
$$

being $f$ the interface. Using Darcy's Law we get

$$
\operatorname{curl} \operatorname{curl} v=\left(-\partial_{x_{1}} \partial_{x_{3}} \rho,-\partial_{x_{2}} \partial_{x_{3}} \rho,\left(\partial_{x_{1}}^{2}+\partial_{x_{2}}^{2}\right) \rho\right) .
$$

Since $\operatorname{div} v=0$ we have $\operatorname{curl} \operatorname{curl} v=-\Delta v$, therefore it follows

$$
v=\left(\partial_{x_{1}} \Delta^{-1} \partial_{x_{3}} \rho, \partial_{x_{2}} \Delta^{-1} \partial_{x_{3}} \rho,-\left(\partial_{x_{1}}^{2}+\partial_{x_{2}}^{2}\right) \Delta^{-1} \rho\right) .
$$

The integral operators $\partial_{x_{1}} \Delta^{-1}$ and $\partial_{x_{2}} \Delta^{-1}$ are given by the kernels

$$
K_{1}\left(x_{1}, x_{2}, x_{3}\right)=\frac{1}{4 \pi} \frac{x_{1}}{\left(x_{1}^{2}+x_{2}^{2}+x_{3}^{2}\right)^{3 / 2}}, \quad K_{2}\left(x_{1}, x_{2}, x_{3}\right)=\frac{1}{4 \pi} \frac{x_{2}}{\left(x_{1}^{2}+x_{2}^{2}+x_{3}^{2}\right)^{3 / 2}},
$$

respectively, thus the velocity can be expressed by

$$
v=\left(K_{1} * \partial_{x_{3}} \rho, K_{2} * \partial_{x_{3}} \rho,-K_{1} * \partial_{x_{1}} \rho-K_{2} * \partial_{x_{2}} \rho\right) .
$$

Since $\rho$ satisfies (4) we have

$$
\nabla \rho=\left(\rho_{2}-\rho_{1}\right)\left(\partial_{x_{1}} f\left(x_{1}, x_{2}, t\right), \partial_{x_{2}} f\left(x_{1}, x_{2}, t\right),-1\right) \delta\left(x_{3}-f\left(x_{1}, x_{2}, t\right)\right),
$$


where $\delta$ is the Dirac distribution. Using (6) we obtain

$$
v\left(x_{1}, x_{2}, x_{3}, t\right)=-\frac{\rho_{2}-\rho_{1}}{4 \pi} P V \int_{\mathbb{R}^{2}} \frac{\left(y_{1}, y_{2}, \nabla f(x-y, t) \cdot y\right)}{\left[|y|^{2}+\left(x_{3}-f(x-y, t)\right)^{2}\right]^{3 / 2}} d y,
$$

where we note $x=\left(x_{1}, x_{2}\right), y=\left(y_{1}, y_{2}\right)$ and $\nabla f(x-y, t) \cdot y=\partial_{x_{1}} f(x-y, t) y_{1}+\partial_{x_{2}} f(x-y, t) y_{2}$. In $(8) x_{3} \neq f(x, t)$ and the principal value is taken at infinity (see [21]). When $x_{3}$ approaches $f(x, t)$ in the normal direction, we get a discontinuity on the velocity due to the fact that the vorticity is concentrated on the interface. Thus, for $\varepsilon>0$ we define

$$
v^{1}(x, f(x, t), t)=\lim _{\varepsilon \rightarrow 0} v\left(x_{1}-\varepsilon \partial_{x_{1}} f(x, t), x_{2}-\varepsilon \partial_{x_{2}} f(x, t), f(x, t)+\varepsilon, t\right),
$$

and

$$
v^{2}(x, f(x, t), t)=\lim _{\varepsilon \rightarrow 0} v\left(x_{1}+\varepsilon \partial_{x_{1}} f(x, t), x_{2}+\varepsilon \partial_{x_{2}} f(x, t), f(x, t)-\varepsilon, t\right) .
$$

It follows

$$
\begin{aligned}
v^{1}(x, f(x, t), t)= & -\frac{\rho_{2}-\rho_{1}}{4 \pi} P V \int_{\mathbb{R}^{2}} \frac{\left(y_{1}, y_{2}, \nabla f(x-y, t) \cdot y\right)}{\left[|y|^{2}+(f(x, t)-f(x-y, t))^{2}\right]^{3 / 2}} d y \\
& +\frac{\rho_{2}-\rho_{1}}{2} \frac{\partial_{x_{1}} f(x, t)\left(1,0, \partial_{x_{1}} f(x, t)\right)}{1+\left(\partial_{x_{1}} f(x, t)\right)^{2}+\left(\partial_{x_{2}} f(x, t)\right)^{2}} \\
& +\frac{\rho_{2}-\rho_{1}}{2} \frac{\partial_{x_{2}} f(x, t)\left(0,1, \partial_{x_{2}} f(x, t)\right)}{1+\left(\partial_{x_{1}} f(x, t)\right)^{2}+\left(\partial_{x_{2}} f(x, t)\right)^{2}}, \\
v^{2}(x, f(x, t), t)= & -\frac{\rho_{2}-\rho_{1}}{4 \pi} P V \int_{\mathbb{R}^{2}} \frac{\left(y_{1}, y_{2}, \nabla f(x-y, t) \cdot y\right)}{\left[|y|^{2}+(f(x, t)-f(x-y, t))^{2}\right]^{3 / 2}} d y \\
& -\frac{\rho_{2}-\rho_{1}}{2} \frac{\partial_{x_{1}} f(x, t)\left(1,0, \partial_{x_{1}} f(x, t)\right)}{1+\left(\partial_{x_{1}} f(x, t)\right)^{2}+\left(\partial_{x_{2}} f(x, t)\right)^{2}} \\
& -\frac{\rho_{2}-\rho_{1}}{2} \frac{\partial_{x_{2}} f(x, t)\left(0,1, \partial_{x_{2}} f(x, t)\right)}{1+\left(\partial_{x_{1}} f(x, t)\right)^{2}+\left(\partial_{x_{2}} f(x, t)\right)^{2}} .
\end{aligned}
$$

The velocity in the tangential directions only moves the particles on the surface $f(x, t)$; i.e., if we rewrite the velocity in the tangential directions, we only make a change on the parametrization and do not alter the shape of the interface. Thus, it follows that

$$
v(x, f(x, t), t)=-\frac{\rho_{2}-\rho_{1}}{4 \pi} P V \int_{\mathbb{R}^{2}} \frac{\left(y_{1}, y_{2}, \nabla f(x-y, t) \cdot y\right)}{\left[|y|^{2}+(f(x, t)-f(x-y, t))^{2}\right]^{3 / 2}} d y,
$$

due to the fact that the terms

$$
\begin{aligned}
& \pm \frac{\rho_{2}-\rho_{1}}{2} \frac{\partial_{x_{1}} f(x, t)\left(1,0, \partial_{x_{1}} f(x, t)\right)}{1+\left(\partial_{x_{1}} f(x, t)\right)^{2}+\left(\partial_{x_{2}} f(x, t)\right)^{2}}, \\
& \pm \frac{\rho_{2}-\rho_{1}}{2} \frac{\partial_{x_{2}} f(x, t)\left(0,1, \partial_{x_{2}} f(x, t)\right)}{1+\left(\partial_{x_{1}} f(x, t)\right)^{2}+\left(\partial_{x_{2}} f(x, t)\right)^{2}},
\end{aligned}
$$

are in the tangential directions. Moreover, if we add the following tangential terms to (11)

$$
\frac{\rho_{2}-\rho_{1}}{4 \pi} P V \int_{\mathbb{R}^{2}} \frac{y_{1}}{\left[|y|^{2}+(f(x, t)-f(x-y, t))^{2}\right]^{3 / 2}} d y\left(1,0, \partial_{x_{1}} f(x, t)\right),
$$




$$
\frac{\rho_{2}-\rho_{1}}{4 \pi} P V \int_{\mathbb{R}^{2}} \frac{y_{2}}{\left[|y|^{2}+(f(x, t)-f(x-y, t))^{2}\right]^{3 / 2}} d y\left(0,1, \partial_{x_{2}} f(x, t)\right)
$$

we obtain

$$
v(x, f(x, t), t)=\frac{\rho_{2}-\rho_{1}}{4 \pi}\left(0,0, P V \int_{\mathbb{R}^{2}} \frac{(\nabla f(x, t)-\nabla f(x-y, t)) \cdot y}{\left[|y|^{2}+(f(x, t)-f(x-y, t))^{2}\right]^{3 / 2}} d y\right) .
$$

Finally we have the contour equation given by

$$
\begin{aligned}
\frac{d f}{d t}(x, t) & =\frac{\rho_{2}-\rho_{1}}{4 \pi} P V \int_{\mathbb{R}^{2}} \frac{(\nabla f(x, t)-\nabla f(x-y, t)) \cdot y}{\left[|y|^{2}+(f(x, t)-f(x-y, t))^{2}\right]^{3 / 2}} d y, \\
f(x, 0) & =f_{0}(x) .
\end{aligned}
$$

In the periodic case, we can obtain an equivalent equation to (13) due to the integral operators $\partial_{x_{1}} \Delta^{-1}$ and $\partial_{x_{2}} \Delta^{-1}$ can be presented by the kernels

$$
\begin{aligned}
& K_{1}^{p}\left(x_{1}, x_{2}, x_{3}\right)=\frac{1}{4 \pi}\left(\frac{x_{1}}{\left(x_{1}^{2}+x_{2}^{2}+x_{3}^{2}\right)^{3 / 2}} L\left(x_{1}, x_{2}, x_{3}\right)+M\left(x_{1}, x_{2}, x_{3}\right)\right), \\
& K_{2}^{p}\left(x_{1}, x_{2}, x_{3}\right)=\frac{1}{4 \pi}\left(\frac{x_{2}}{\left(x_{1}^{2}+x_{2}^{2}+x_{3}^{2}\right)^{3 / 2}} L\left(x_{1}, x_{2}, x_{3}\right)+M\left(x_{1}, x_{2}, x_{3}\right)\right),
\end{aligned}
$$

respectively for $\left(x_{1}, x_{2}, x_{3}\right) \in \mathbb{T}^{2} \times \mathbb{R}$ with $\mathbb{T}^{2}=[-\pi, \pi]^{2}$ and the functions $L, M \in C^{\infty}\left(\mathbb{T}^{2} \times \mathbb{R}\right)$ (see [20] for the kernel of the Riesz potentials on the n-torus). Adding an appropiate function to the singular part of $K_{1}^{p}$ and $K_{2}^{P}$, we can choose

$$
\begin{aligned}
& L \in C_{c}^{\infty}\left(\mathbb{T}^{2} \times \mathbb{R}\right), \quad L \geq 0, \quad \operatorname{supp} L \subset\left\{x_{1}^{2}+x_{2}^{2}+x_{3}^{2} \leq 4\right\}, \\
& L=1 \text { in }\left\{x_{1}^{2}+x_{2}^{2}+x_{3}^{2} \leq 1\right\} \quad \text { and } \quad L\left(-x_{1},-x_{2},-x_{3}\right)=L\left(x_{1}, x_{2}, x_{3}\right) .
\end{aligned}
$$

The function $M$ belongs to $C_{b}^{\infty}\left(\mathbb{T}^{2} \times \mathbb{R}\right)$ and $M(0,0,0)=0$. The velocity can be expressed by

$$
v=\left(K_{1}^{p} * \partial_{x_{3}} \rho, K_{2}^{p} * \partial_{x_{3}} \rho,-K_{1}^{p} * \partial_{x_{1}} \rho-K_{2}^{p} * \partial_{x_{2}} \rho\right),
$$

and due to (7) it follows (suppressing the dependence on t)

$$
\begin{aligned}
v\left(x_{1}, x_{2}, x_{3}\right)= & -\frac{\rho_{2}-\rho_{1}}{4 \pi} P V \int_{\mathbb{T}^{2}} \frac{\left(y_{1}, y_{2}, \nabla f(x-y) \cdot y\right)}{\left[|y|^{2}+\left(x_{3}-f(x-y)\right)^{2}\right]^{3 / 2}} L\left(y, x_{3}-f(x-y)\right) d y \\
& -\frac{\rho_{2}-\rho_{1}}{4 \pi} \int_{\mathbb{T}^{2}}(1,1, \nabla f(x-y) \cdot(1,1)) M\left(y, x_{3}-f(x-y)\right) d y,
\end{aligned}
$$

if $x_{3} \neq f(x)$. Adding a term in the tangential direction we obtain

$$
\begin{aligned}
v(x, f(x))=\frac{\rho_{2}-\rho_{1}}{4 \pi}(0,0 & , \int_{\mathbb{T}^{2}} \frac{(\nabla f(x)-\nabla f(x-y)) \cdot y}{\left[|y|^{2}+(f(x)-f(x-y))^{2}\right]^{3 / 2}} L(y, f(x)-f(x-y)) d y \\
& \left.\left.+\int_{\mathbb{T}^{2}}(\nabla f(x)-\nabla f(x-y)) \cdot(1,1)\right) M(y, f(x)-f(x-y)) d y\right) .
\end{aligned}
$$

Finally we have the contour equation in the periodic case given by

$$
\begin{aligned}
\frac{d f}{d t}(x, t)= & \frac{\rho_{2}-\rho_{1}}{4 \pi} \int_{\mathbb{T}^{2}} \frac{(\nabla f(x, t)-\nabla f(x-y, t)) \cdot y}{\left[|y|^{2}+(f(x, t)-f(x-y, t))^{2}\right]^{3 / 2}} L(y, f(x, t)-f(x-y, t)) d y \\
& \left.+\frac{\rho_{2}-\rho_{1}}{4 \pi} \int_{\mathbb{T}^{2}}(\nabla f(x, t)-\nabla f(x-y, t)) \cdot(1,1)\right) M(y, f(x, t)-f(x-y, t)) d y, \\
f(x, 0)= & f_{0}(x) .
\end{aligned}
$$


We use both formulations throughout the paper. Suppose that the function $f(x)$ only depends on $x_{1}$ in equation (13). Then the contour equation in the 2-D case (with a 1-D interface) follows

$$
\begin{aligned}
\frac{d f}{d t}(x, t) & =\frac{\rho_{2}-\rho_{1}}{2 \pi} P V \int_{\mathbb{R}} \frac{\left(\partial_{x} f(x, t)-\partial_{x} f(x-\alpha, t)\right) \alpha}{\alpha^{2}+(f(x, t)-f(x-\alpha, t))^{2}} d \alpha, \\
f(x, 0) & =f_{0}(x) ; \quad x \in \mathbb{R} .
\end{aligned}
$$

This equation can be obtained in a similar way that (13) using the stream function. Performing a two-dimensional analysis using the stream function, we obtain an equivalent equation to (16) in the two dimensional periodic case as follows

$$
\begin{aligned}
\frac{d f}{d t}(x, t)= & \frac{\rho_{2}-\rho_{1}}{2 \pi} \int_{\mathbb{T}} \frac{\left(\partial_{x} f(x, t)-\partial_{x} f(x-\alpha, t)\right) \alpha}{\alpha^{2}+(f(x, t)-f(x-\alpha, t))^{2}} P(\alpha, f(x, t)-f(x-\alpha, t)) d \alpha \\
& +\frac{\rho_{2}-\rho_{1}}{2 \pi} \int_{\mathbb{T}}\left(\partial_{x} f(x, t)-\partial_{x} f(x-\alpha, t)\right) Q(\alpha, f(x, t)-f(x-\alpha, t)) d \alpha \\
f(x, 0)= & f_{0}(x),
\end{aligned}
$$

with

$$
\begin{aligned}
& P\left(x_{1}, x_{2}\right) \in C_{c}^{\infty}(\mathbb{T} \times \mathbb{R}), \quad P \geq 0, \quad \operatorname{supp} P \subset\left\{x_{1}^{2}+x_{2}^{2} \leq 4\right\}, \\
& P=1 \text { in }\left\{x_{1}^{2}+x_{2}^{2} \leq 1\right\} \quad \text { and } \quad P\left(-x_{1},-x_{2}\right)=P\left(x_{1}, x_{2}\right) .
\end{aligned}
$$

The function $Q\left(x_{1}, x_{2}\right)$ belongs to $C_{b}^{\infty}(\mathbb{T} \times \mathbb{R})$ and $Q(0,0)=0$.

If we consider the linearized equation of the motion, we obtain a dissipative equation when $\rho_{1}<\rho_{2}$ (the greater density is below) and an unstable equation when $\rho_{1}>\rho_{2}$. The unstable linearized equation presents an instability similar to the Kelvin-Helmholtz's (see [6]).

As usual, we note the Riesz transforms in $\mathbb{R}^{2}$ (see [21])

$$
\begin{aligned}
& R_{1} f(x)=\frac{1}{2 \pi} P . V \cdot \int_{\mathbb{R}^{2}} \frac{y_{1}}{|y|^{3}} f(x-y) d y, \\
& R_{2} f(x)=\frac{1}{2 \pi} P . V \cdot \int_{\mathbb{R}^{2}} \frac{y_{2}}{|y|^{3}} f(x-y) d y,
\end{aligned}
$$

and the operator $\Lambda^{s} f$ defined by the fourier transform $\widehat{\Lambda^{s} f}(\xi)=|\xi|^{s} \widehat{f}(\xi)$.

Suppose that $f(x)$ is uniformly small and we can neglect the terms of order grater than one in (13), then it reduces to the following linear equation

$$
\begin{aligned}
& f_{t}=\frac{\rho_{1}-\rho_{2}}{2}\left(R_{1} \partial_{x_{1}} f+R_{2} \partial_{x_{2}} f\right)=\frac{\rho_{1}-\rho_{2}}{2} \Lambda f, \\
& f(x, 0)=f_{0}(x) .
\end{aligned}
$$

Applying the fourier transform we get

$$
\hat{f}(\xi)=\hat{f}_{0}(\xi) e^{\frac{\rho_{1}-\rho_{2}}{2}|\xi| t},
$$

and therefore (18) is a dissipative equation when $\rho_{1}<\rho_{2}$ and an ill posed problem in the case $\rho_{1}>\rho_{2}$ with a general initial data in the Schwartz class. We need an analytic initial data in order to get a well posed problem for $\rho_{1}>\rho_{2}$. 


\section{The conservation of mass equation}

We show that if $\rho$ is defined by (4) and $f(x, t)$ is convected by the velocity (12) then $\rho$ is a weak solution of the conservation of mass equation (3) and conversely. From now on, $\Omega$ is equal to $\mathbb{R}^{2}$ or $\mathbb{T}^{2}$ and $\widetilde{x}=\left(x_{1}, x_{2}, x_{3}\right)$.

Definition 3.1 The density $\rho$ is a weak solution of the conservation of mass equation if for any $\varphi \in C^{\infty}(\Omega \times \mathbb{R} \times(0, T)), \varphi$ with compact support in the real case and periodic in $\left(x_{1}, x_{2}\right)$ otherwise, we have

$$
\int_{0}^{T} \int_{\Omega} \int_{\mathbb{R}}\left(\rho(\widetilde{x}, t) \partial_{t} \varphi(\widetilde{x}, t)+v(\widetilde{x}, t) \rho(\widetilde{x}, t) \nabla \varphi(\widetilde{x}, t)\right) d \widetilde{x} d t=0,
$$

where the incompressible velocity $v$ is given by Darcy's law.

Then

Proposition 3.2 If $f(x, t)$ satisfies (13) and $\rho(\widetilde{x}, t)$ is defined by (4), then $\rho$ is a weak solution of the conservation of mass equation. Furthermore, if $\rho$ is a weak solution of the conservation of mass equation given by (4), then $f(x, t)$ satisfies (13).

Proof: Let $\rho$ be a weak solution of (3) defined by (4). Integrating by parts we have

$$
\begin{aligned}
I & =\int_{0}^{T} \int_{\Omega} \int_{\mathbb{R}} \rho \partial_{t} \varphi d \widetilde{x} d t=\rho_{1} \int_{0}^{T} \int_{\left\{x_{3}>f\right\}} \partial_{t} \varphi d \widetilde{x} d t+\rho_{2} \int_{0}^{T} \int_{\left\{x_{3}<f\right\}} \partial_{t} \varphi d \widetilde{x} d t \\
& =\left(\rho_{1}-\rho_{2}\right) \int_{0}^{T} \int_{\Omega} \varphi(x, f(x, t), t) \partial_{t} f(x, t) d x d t .
\end{aligned}
$$

On the other hand, due to (9) and (10) we obtain

$$
\begin{aligned}
J & =\int_{0}^{T} \int_{\Omega} \int_{\mathbb{R}} \rho v \nabla \varphi d \widetilde{x} d t=\rho_{1} \int_{0}^{T} \int_{\left\{x_{3}>f\right\}} v \nabla \varphi d \widetilde{x} d t+\rho_{2} \int_{0}^{T} \int_{\left\{x_{3}<f\right\}} v \nabla \varphi d \widetilde{x} d t \\
& =\int_{0}^{T} \int_{\Omega} \varphi(x, f(x, t), t)\left(\rho_{1} v^{1}(x, f(x, t), t)-\rho_{2} v^{2}(x, f(x, t), t)\right) \cdot\left(\partial_{x_{1}} f(x, t), \partial_{x_{2}} f(x, t),-1\right) d x d t \\
& =\left(\rho_{1}-\rho_{2}\right) \int_{0}^{T} \int_{\Omega} \varphi(x, f(x, t), t) v(x, f(x, t), t) \cdot\left(\partial_{x_{1}} f(x, t), \partial_{x_{2}} f(x, t),-1\right) d x d t,
\end{aligned}
$$

where $v(x, f(x, t), t)$ is given by (11). We get

$$
J=\frac{\left(\rho_{1}-\rho_{2}\right)^{2}}{4 \pi} \int_{0}^{T} \int_{\Omega} \varphi(x, f(x, t), t) P V \int_{\mathbb{R}^{2}} \frac{(\nabla f(x, t)-\nabla f(x-y, t)) \cdot y}{\left[|y|^{2}+(f(x, t)-f(x-y, t))^{2}\right]^{3 / 2}} d y d x d t .
$$

Then $I+J=0$ due to (19). Thus, if we choose $\varphi(\widetilde{x}, t)=\varphi(x, t)$ for $x_{3} \in\left[-\|f\|_{L^{\infty}},\|f\|_{L^{\infty}}\right]$ follows that $f(x, t)$ fulfils $(13)$.

Following the same arguments it is easy to check that if $f(x, t)$ satisfies (13), then $\rho$ is a weak solution given by (4).

Remark 3.3 Note that due to (5), the velocity satisfies

$$
v=\left(R_{1}\left(R_{3} \rho\right), R_{2}\left(R_{3} \rho\right),-\left(R_{1}^{2}+R_{2}^{2}\right)(\rho)\right),
$$

where the operators $R_{1}, R_{2}$ and $R_{3}$ are the Riesz transforms in three dimensions (see [21]). Since $\rho \in L^{\infty}(\Omega \times \mathbb{R})$ then $v$ belongs to BMO (bounded mean oscillation) and therefore $v$ is in $L^{2}(\Omega \times \mathbb{R})$ locally (see [21] for the definitions and properties of the BMO space). 


\section{Local well-posedness for the stable case}

In this section we prove local existence and uniqueness for the stable case using energy estimates. First we study the case $\Omega=\mathbb{R}^{2}$ and at the end of the section we give the main differences with the periodic domain. Denote the Sobolev spaces by $H^{k}$, the Holder spaces by $C^{k, \delta}$ with $0 \leq \delta<1$ the Holder continuity and the hessian matrix of a function $f(x)$ by $\nabla^{2} f(x)$. The norms of $\bar{H}^{k}$ and $C^{k, \delta}$ are defined as follows

$$
\begin{gathered}
\|f\|_{H^{k}}^{2}=\|f\|_{L^{2}}^{2}+\left\|\Lambda^{k} f\right\|_{L^{2}}^{2} \\
\|f\|_{C^{k, \delta}}=\|f\|_{C^{k}}+\max _{i+j=k} \max _{x \neq y} \frac{\left|\partial_{x_{1}}^{i} \partial_{x_{2}}^{j} f(x)-\partial_{x_{1}}^{i} \partial_{x_{2}}^{j} f(y)\right|}{|x-y|^{\delta}} .
\end{gathered}
$$

\subsection{Case $\Omega=\mathbb{R}^{2}$}

The main theorem in this section is the following

Theorem 4.1 Let $f_{0}(x) \in H^{k}\left(\mathbb{R}^{2}\right)$ for $k \geq 4$ and $\rho_{2}>\rho_{1}$. Then there exists a time $T>0$ so that there is a unique solution to (13) in $C^{1}\left([0, T] ; H^{k}\left(\mathbb{R}^{2}\right)\right)$ with $f(x, 0)=f_{0}(x)$.

We choose $\rho_{2}-\rho_{1}=4 \pi$ without loss of generality, then

$$
\begin{aligned}
\frac{d f}{d t}(x, t) & =P V \int_{\mathbb{R}^{2}} \frac{(\nabla f(x, t)-\nabla f(x-y, t)) \cdot y}{\left[|y|^{2}+(f(x, t)-f(x-y, t))^{2}\right]^{3 / 2}} d y, \\
f(x, 0) & =f_{0}(x) .
\end{aligned}
$$

We show the proof with $k=4$ being analogous for $k>4$. We apply energy methods (see [5] for more details). Then

$$
\begin{aligned}
\frac{1}{2} \frac{d}{d t}\|f\|_{L^{2}}^{2}(t)= & \int_{\mathbb{R}^{2}} f(x) P V \int_{\mathbb{R}^{2}} \frac{(\nabla f(x)-\nabla f(x-y)) \cdot y}{\left[|y|^{2}+(f(x)-f(x-y))^{2}\right]^{3 / 2}} d y d x \\
= & \int_{\mathbb{R}^{2}} f(x) \int_{|y|<1} \frac{(\nabla f(x)-\nabla f(x-y)) \cdot y}{\left[|y|^{2}+(f(x)-f(x-y))^{2}\right]^{3 / 2}} d y d x \\
& +\int_{\mathbb{R}^{2}} f(x) P V \int_{|y|>1} \frac{\nabla f(x) \cdot y}{\left[|y|^{2}+(f(x)-f(x-y))^{2}\right]^{3 / 2}} d y d x \\
& -\int_{\mathbb{R}^{2}} f(x) P V \int_{|y|>1} \frac{\nabla f(x-y) \cdot y}{\left[|y|^{2}+(f(x)-f(x-y))^{2}\right]^{3 / 2}} d y d x \\
= & I_{1}+I_{2}+I_{3} .
\end{aligned}
$$

The identity

$$
\partial_{x_{i}} f(x)-\partial_{x_{i}} f(x-y)=\int_{0}^{1} \nabla \partial_{x_{i}} f(x+(s-1) y) \cdot y d s
$$

yields

$$
\begin{aligned}
I_{1} & \leq C \int_{0}^{1} d s \int_{|y|<1}|y|^{-1} \int_{\mathbb{R}^{2}} \frac{\left|f(x) \| \nabla^{2} f(x+(s-1) y)\right|}{\left[1+\left((f(x)-f(x-y))^{2}|y|^{-2}\right]^{3 / 2}\right.} d x d y \\
& \leq C \int_{0}^{1} d s \int_{|y|<1}|y|^{-1} d y\|f\|_{L^{2}} \sum_{i+j=2}\left\|\partial_{x_{1}}^{i} \partial_{x_{2}}^{j} f\right\|_{L^{2}} \leq C\|f\|_{H^{2}}^{2} .
\end{aligned}
$$


Integrating by parts, the term $I_{2}$ is written

$$
\begin{aligned}
I_{2} & =\frac{3}{2} \int_{|y|>1} \int_{\mathbb{R}^{2}}|f(x)|^{2} \frac{(f(x)-f(x-y))(\nabla f(x)-\nabla f(x-y)) \cdot y}{\left[|y|^{2}+\left((f(x)-f(x-y))^{2}\right]^{5 / 2}\right.} d x d y \\
& \leq C \int_{|y|>1}|y|^{-3} \int_{\mathbb{R}^{2}}|f(x)|^{2} \frac{|f(x)-f(x-y)||y|^{-1}|\nabla f(x)-\nabla f(x-y)|}{\left[1+\left((f(x)-f(x-y))^{2}|y|^{-2}\right]^{5 / 2}\right.} d x d y \\
& \leq C\|f\|_{L^{\infty}}\|f\|_{H^{1}}^{2} .
\end{aligned}
$$

Integrating by parts in $I_{3}$, it follows

$$
\begin{aligned}
I_{3}= & \int_{|y|>1} \int_{\mathbb{R}^{2}} f(x) f(x-y) \frac{|y|^{2}-2(f(x)-f(x-y))^{2}}{\left[|y|^{2}+(f(x)-f(x-y))^{2}\right]^{5 / 2}} d x d y \\
& +3 \int_{|y|>1} \int_{\mathbb{R}^{2}} f(x) f(x-y) \frac{(f(x)-f(x-y)) \nabla f(x-y) \cdot y}{\left[|y|^{2}+(f(x)-f(x-y))^{2}\right]^{5 / 2}} d x d y \\
& -\int_{|y|=1} \int_{\mathbb{R}^{2}} f(x) f(x-y) \frac{|y|^{2}}{\left[|y|^{2}+(f(x)-f(x-y))^{2}\right]^{3 / 2}} d x d \sigma(y) \\
\leq & C\left(\|f\|_{L^{\infty}}+1\right)\|f\|_{H^{1}}^{2} .
\end{aligned}
$$

Using Sobolev inequalities, we get finally

$$
\frac{d}{d t}\|f\|_{L^{2}}^{2}(t) \leq C\left(\|f\|_{H^{2}}^{3}(t)+1\right) .
$$

We consider the quantity

$$
\frac{1}{2} \frac{d}{d t}\left\|\partial_{x_{1}}^{4} f\right\|_{L^{2}}^{2}(t)=I_{4}+I_{5}+I_{6}+I_{7}+I_{8}
$$

where

$$
\begin{aligned}
& I_{4}=\int_{\mathbb{R}^{2}} \partial_{x_{1}}^{4} f(x) P V \int_{\mathbb{R}^{2}} \frac{\left(\nabla \partial_{x_{1}}^{4} f(x)-\nabla \partial_{x_{1}}^{4} f(x-y)\right) \cdot y}{\left[|y|^{2}+(f(x)-f(x-y))^{2}\right]^{3 / 2}} d y d x, \\
& I_{5}=4 \int_{\mathbb{R}^{2}} \partial_{x_{1}}^{4} f(x) \int_{\mathbb{R}^{2}}\left(\nabla \partial_{x_{1}}^{3} f(x)-\nabla \partial_{x_{1}}^{3} f(x-y)\right) \cdot y \partial_{x_{1}} A(x, y) d y d x, \\
& I_{6}=6 \int_{\mathbb{R}^{2}} \partial_{x_{1}}^{4} f(x) \int_{\mathbb{R}^{2}}\left(\nabla \partial_{x_{1}}^{2} f(x)-\nabla \partial_{x_{1}}^{2} f(x-y)\right) \cdot y \partial_{x_{1}}^{2} A(x, y) d y d x, \\
& I_{7}=4 \int_{\mathbb{R}^{2}} \partial_{x_{1}}^{4} f(x) \int_{\mathbb{R}^{2}}\left(\nabla \partial_{x_{1}} f(x)-\nabla \partial_{x_{1}} f(x-y)\right) \cdot y \partial_{x_{1}}^{3} A(x, y) d y d x, \\
& I_{8}=\int_{\mathbb{R}^{2}} \partial_{x_{1}}^{4} f(x) \int_{\mathbb{R}^{2}}(\nabla f(x)-\nabla f(x-y)) \cdot y \partial_{x_{1}}^{4} A(x, y) d y d x,
\end{aligned}
$$

and

$$
A(x, y)=\left[|y|^{2}+(f(x)-f(x-y))^{2}\right]^{-3 / 2} .
$$

The most singular term is $I_{4}$. In order to estimate it

$$
\begin{aligned}
I_{4}= & \int_{\mathbb{R}^{2}} \partial_{x_{1}}^{4} f(x) P V \int_{\mathbb{R}^{2}} \frac{\nabla \partial_{x_{1}}^{4} f(x, t) \cdot y}{\left[|y|^{2}+(f(x, t)-f(x-y, t))^{2}\right]^{3 / 2}} d y d x \\
& -\int_{\mathbb{R}^{2}} \partial_{x_{1}}^{4} f(x) P V \int_{\mathbb{R}^{2}} \frac{\nabla \partial_{x_{1}}^{4} f(y, t) \cdot(x-y)}{\left[|x-y|^{2}+(f(x, t)-f(y, t))^{2}\right]^{3 / 2}} d y d x \\
= & J_{1}+J_{2} .
\end{aligned}
$$


Integrating by parts

$$
\begin{aligned}
J_{1} & =\frac{3}{2} \int_{\mathbb{R}^{2}}\left|\partial_{x_{1}}^{4} f(x)\right|^{2} P V \int_{\mathbb{R}^{2}} \frac{(f(x)-f(x-y))(\nabla f(x)-\nabla f(x-y)) \cdot y}{\left[|y|^{2}+\left((f(x)-f(x-y))^{2}\right]^{5 / 2}\right.} d y d x \\
& =\frac{3}{2} \int_{\mathbb{R}^{2}}\left|\partial_{x_{1}}^{4} f(x)\right|^{2}\left(\int_{|y|>1} d y+P V \int_{|y|<1} d y\right) d x \\
& \leq \frac{3}{2}\|f\|_{C^{1}}\left\|\partial_{x_{1}}^{4} f\right\|_{L^{2}}^{2}+\frac{3}{2} M(f)\left\|\partial_{x_{1}}^{4} f\right\|_{L^{2}}^{2},
\end{aligned}
$$

where

$$
M(f)=\max _{x}\left|P V \int_{|y|<1} \frac{(f(x)-f(x-y))(\nabla f(x)-\nabla f(x-y)) \cdot y}{\left[|y|^{2}+\left((f(x)-f(x-y))^{2}\right]^{5 / 2}\right.} d y\right| .
$$

We estimate this maximum in the following form

$$
\begin{aligned}
M(f) \leq & \max _{x}\left|\int_{|y|<1} \frac{(f(x)-f(x-y)-\nabla f(x) \cdot y)(\nabla f(x)-\nabla f(x-y)) \cdot y}{\left[|y|^{2}+\left((f(x)-f(x-y))^{2}\right]^{5 / 2}\right.} d y\right| \\
& +\max _{x}\left|\int_{|y|<1} \frac{(\nabla f(x) \cdot y)\left((\nabla f(x)-\nabla f(x-y)) \cdot y-y \cdot \nabla^{2} f(x) \cdot y\right)}{\left[|y|^{2}+\left((f(x)-f(x-y))^{2}\right]^{5 / 2}\right.} d y\right| \\
& +\max _{x}\left|\int_{|y|<1}(\nabla f(x) \cdot y)\left(y \cdot \nabla^{2} f(x) \cdot y\right)(B(x, y)-C(x, y)) d y\right| \\
& +\max _{x}\left|P V \int_{|y|<1} \frac{(\nabla f(x) \cdot y)\left(y \cdot \nabla^{2} f(x) \cdot y\right)}{\left[|y|^{2}+(\nabla f(x) \cdot y)^{2}\right]^{5 / 2}} d y\right|,
\end{aligned}
$$

where

$$
B(x, y)=\left[|y|^{2}+\left((f(x)-f(x-y))^{2}\right]^{-5 / 2}, \quad C(x, y)=\left[|y|^{2}+(\nabla f(x) \cdot y)^{2}\right]^{-5 / 2} .\right.
$$

Making the change of variables $y=-z$, we obtain that the last integral in (23) is null, then we can estimate $M(f)$ by

$$
\begin{aligned}
M(f) \leq & \|f\|_{C^{2}}^{2} \max _{x}\left|\int_{|y|<1} \frac{|y|^{-1}}{\left[1+\left((f(x)-f(x-y))|y|^{-1}\right)^{2}\right]^{5 / 2}} d y\right| \\
& +\left.\|f\|_{C^{1}}\|f\|_{C^{2, \delta}}\left|\int_{|y|<1}\right| y\right|^{-2+\delta} d y\left|+\|f\|_{C^{1}}^{2}\|f\|_{C^{2}}^{2}\right| \int_{|y|<1}|y|^{-1} d y \mid \\
\leq & C\left(\|f\|_{C^{2}}^{2}+\|f\|_{C^{1}}\|f\|_{C^{2, \delta}}+\|f\|_{C^{1}}^{2}\|f\|_{C^{2}}^{2}\right),
\end{aligned}
$$

with $0<\delta<1$, having finally

$$
J_{1} \leq C\left(\|f\|_{C^{2, \delta}}^{4}+1\right)\left\|\partial_{x_{1}}^{4} f\right\|_{L^{2}}^{2} .
$$

In order to estimate $J_{2}$, we integrate by parts getting

$$
\begin{aligned}
J_{2} & =\int_{\mathbb{R}^{2}} \partial_{x_{1}}^{4} f(x) P V \int_{\mathbb{R}^{2}} \frac{\nabla_{y}\left(\partial_{x_{1}}^{4} f(x)-\partial_{x_{1}}^{4} f(y)\right) \cdot(x-y)}{\left[|x-y|^{2}+(f(x)-f(y))^{2}\right]^{3 / 2}} d y d x \\
& =K_{1}+K_{2}
\end{aligned}
$$

with

$$
K_{1}=-\int_{\mathbb{R}^{2}} \partial_{x_{1}}^{4} f(x) P V \int_{\mathbb{R}^{2}} \frac{\partial_{x_{1}}^{4} f(x)-\partial_{x_{1}}^{4} f(y)}{\left[|x-y|^{2}+(f(x)-f(y))^{2}\right]^{3 / 2}} d y d x
$$


and

$$
K_{2}=\int_{\mathbb{R}^{2}} \partial_{x_{1}}^{4} f(x) \int_{\mathbb{R}^{2}}\left(\partial_{x_{1}}^{4} f(x)-\partial_{x_{1}}^{4} f(y)\right) \frac{3(f(x)-f(y))(f(x)-f(y)-\nabla f(y) \cdot(x-y))}{\left[|x-y|^{2}+(f(x)-f(y))^{2}\right]^{5 / 2}} d y d x .
$$

Making a change of variables we can obtain

$$
\begin{aligned}
K_{1} & =-P V \int_{\mathbb{R}^{2}} \int_{\mathbb{R}^{2}} \partial_{x_{1}}^{4} f(x) \frac{\partial_{x_{1}}^{4} f(x)-\partial_{x_{1}}^{4} f(y)}{\left[|x-y|^{2}+(f(x)-f(y))^{2}\right]^{3 / 2}} d y d x \\
& =P V \int_{\mathbb{R}^{2}} \int_{\mathbb{R}^{2}} \partial_{x_{1}}^{4} f(y) \frac{\partial_{x_{1}}^{4} f(x)-\partial_{x_{1}}^{4} f(y)}{\left[|x-y|^{2}+(f(x)-f(y))^{2}\right]^{3 / 2}} d y d x \\
& =-\frac{1}{2} \int_{\mathbb{R}^{2}} \int_{\mathbb{R}^{2}} \frac{\left(\partial_{x_{1}}^{4} f(x)-\partial_{x_{1}}^{4} f(y)\right)^{2}}{\left[|x-y|^{2}+(f(x)-f(y))^{2}\right]^{3 / 2}} d y d x \\
& \leq 0 .
\end{aligned}
$$

Here we observe the main difference with the unstable case in which we obtain the opposite sign. Now we consider

$$
K_{2}=L_{1}+L_{2}+L_{3}
$$

being

$$
\begin{gathered}
L_{1}=3 \int_{\mathbb{R}^{2}}\left|\partial_{x_{1}}^{4} f(x)\right|^{2} P V \int_{\mathbb{R}^{2}} \frac{(f(x)-f(y))(f(x)-f(y)-\nabla f(y) \cdot(x-y))}{\left[|x-y|^{2}+(f(x)-f(y))^{2}\right]^{5 / 2}} d y d x, \\
L_{2}=-\frac{3}{4} P V \int_{\mathbb{R}^{2}} \int_{\mathbb{R}^{2}} \partial_{x_{1}}^{4} f(x) \partial_{x_{1}}^{4} f(y) \frac{(f(x)-f(y))(x-y) \cdot\left(\nabla^{2} f(x)+\nabla^{2} f(y)\right) \cdot(x-y)}{\left[|x-y|^{2}+(f(x)-f(y))^{2}\right]^{5 / 2}} d y d x, \\
L_{3}=-3 \int_{\mathbb{R}^{2}} \int_{\mathbb{R}^{2}} \partial_{x_{1}}^{4} f(x) \partial_{x_{1}}^{4} f(y)(f(x)-f(y)) D(x, y) d y d x,
\end{gathered}
$$

with

$$
D(x, y)=\frac{\left(f(x)-f(y)-\nabla f(y) \cdot(x-y)-\frac{1}{4}(x-y) \cdot\left(\nabla^{2} f(x)+\nabla^{2} f(y)\right) \cdot(x-y)\right)}{\left[|x-y|^{2}+(f(x)-f(y))^{2}\right]^{5 / 2}} .
$$

The term $L_{1}$ can be estimated like $J_{1}$ in $(22)$ and one finds that

$$
L_{1} \leq C\left(1+\|f\|_{C^{2, \delta}}^{4}\right)\left\|\partial_{x_{1}}^{4} f\right\|_{L^{2}}^{2} .
$$

Exchanging $x$ for $y$ we obtain that $L_{2}=0$.

For the last term $L_{3}$, it follows

$$
\begin{aligned}
L_{3} \leq & C \int_{\mathbb{R}^{2}}\left|\partial_{x_{1}}^{4} f(x)\right|^{2} \int_{\mathbb{R}^{2}}|f(x)-f(y)||D(x, y)| d y d x \\
& +C \int_{\mathbb{R}^{2}}\left|\partial_{x_{1}}^{4} f(y)\right|^{2} \int_{\mathbb{R}^{2}}|f(x)-f(y)||D(x, y)| d x d y \\
\leq & C \int_{\mathbb{R}^{2}}\left|\partial_{x_{1}}^{4} f(x)\right|^{2} \int_{\mathbb{R}^{2}}|f(x)-f(x-y)||D(x, x-y)| d y d x \\
& +C \int_{\mathbb{R}^{2}}\left|\partial_{x_{1}}^{4} f(y)\right|^{2} \int_{\mathbb{R}^{2}}|f(x+y)-f(y)||D(x+y, y)| d x d y \\
\leq & C \int_{\mathbb{R}^{2}}\left|\partial_{x_{1}}^{4} f(x)\right|^{2} d x\left(\int_{|y|<1} d y+\int_{|y|>1} d y\right)+\int_{\mathbb{R}^{2}}\left|\partial_{x_{1}}^{4} f(y)\right|^{2} d y\left(\int_{|x|<1} d x+\int_{|x|>1} d x\right) \\
\leq & C\|f\|_{C^{1}}\|f\|_{C^{2, \delta}}\left\|\partial_{x_{1}}^{4} f\right\|_{L^{2}}^{2} .
\end{aligned}
$$


Finally

$$
J_{2}=K_{1}+K_{2} \leq K_{2}=L_{1}+L_{2}+L_{3}=L_{1}+L_{3} \leq C\left(\|f\|_{C^{2, \delta}}^{4}+1\right)\left\|\partial_{x_{1}}^{4} f\right\|_{L^{2}}^{2},
$$

and due to (24) we obtain

$$
I_{4} \leq C\left(\|f\|_{C^{2, \delta}}^{4}+1\right)\left\|\partial_{x_{1}}^{4} f\right\|_{L^{2}}^{2} .
$$

Now we estimate the integral $I_{5}$. We have $I_{5}=J_{3}+J_{4}$ where

$$
J_{3}=4 \int_{\mathbb{R}^{2}} \partial_{x_{1}}^{4} f(x) \nabla \partial_{x_{1}}^{3} f(x) \cdot P V \int_{\mathbb{R}^{2}} y \frac{(f(x)-f(x-y))\left(\partial_{x_{1}} f(x)-\partial_{x_{1}} f(x-y)\right)}{\left[|y|^{2}+(f(x)-f(x-y))^{2}\right]^{5 / 2}} d y d x
$$

and

$$
\begin{aligned}
J_{4} & =-4 P V \int_{\mathbb{R}^{2}} \int_{\mathbb{R}^{2}} \partial_{x_{1}}^{4} f(x) \nabla \partial_{x_{1}}^{3} f(y) \cdot(x-y) \frac{(f(x)-f(y))\left(\partial_{x_{1}} f(x)-\partial_{x_{1}} f(y)\right)}{\left[|x-y|^{2}+(f(x)-f(y))^{2}\right]^{5 / 2}} d y d x \\
& =-4 P V \int_{\mathbb{R}^{2}} \int_{\mathbb{R}^{2}} \partial_{x_{1}}^{4} f(x) \partial_{x_{2}} \partial_{x_{1}}^{3} f(y)\left(x_{2}-y_{2}\right) \frac{(f(x)-f(y))\left(\partial_{x_{1}} f(x)-\partial_{x_{1}} f(y)\right)}{\left[|x-y|^{2}+(f(x)-f(y))^{2}\right]^{5 / 2}} d y d x
\end{aligned}
$$

The way to estimate $J_{3}$ is similar to the term $J_{1}$ in (22), and we find that

$$
J_{3} \leq C\left(1+\|f\|_{C^{2, \delta}}^{4}\right)\|f\|_{H^{4}}^{2} .
$$

We decompose the term $J_{4}=K_{3}+K_{4}+K_{5}+K_{6}$ as follows

$$
\begin{gathered}
K_{3}=-4 P V \int_{\mathbb{R}^{2}} \int_{\mathbb{R}^{2}} \partial_{x_{1}}^{4} f(x) \partial_{x_{2}} \partial_{x_{1}}^{3} f(x-y) y_{2} E(x, y) d y d x \\
K_{4}=-4 P V \int_{\mathbb{R}^{2}} \int_{\mathbb{R}^{2}} \partial_{x_{1}}^{4} f(x) \partial_{x_{2}} \partial_{x_{1}}^{3} f(x-y) y_{2} F(x, y) d y d x \\
K_{5}=-4 P V \int_{\mathbb{R}^{2}} \int_{|y|<1} \partial_{x_{1}}^{4} f(x) \partial_{x_{2}} \partial_{x_{1}}^{3} f(x-y) y_{2}(\nabla f(x) \cdot y)\left(\nabla \partial_{x_{1}} f(x) \cdot y\right)(B(x, y)-C(x, y)) d y d x
\end{gathered}
$$

and

$$
K_{6}=-4 P V \int_{\mathbb{R}^{2}} \int_{|y|<1} \partial_{x_{1}}^{4} f(x) \partial_{x_{2}} \partial_{x_{1}}^{3} f(x-y) y_{2} \frac{(\nabla f(x) \cdot y)\left(\nabla \partial_{x_{1}} f(x) \cdot y\right)}{\left[|y|^{2}+(\nabla f(x) \cdot y)^{2}\right]^{5 / 2}} d y d x
$$

where

$$
E(x, y)=\frac{(f(x)-f(x-y)-\nabla f(x) \cdot y)\left(\partial_{x_{1}} f(x)-\partial_{x_{1}} f(x-y)\right)}{\left[|y|^{2}+(f(x)-f(x-y))^{2}\right]^{5 / 2}},
$$

and

$$
F(x, y)=\frac{(\nabla f(x) \cdot y)\left(\partial_{x_{1}} f(x)-\partial_{x_{1}} f(x-y)-\nabla \partial_{x_{1}} f(x) \cdot y \mathcal{X}_{\{|y|<1\}}\right)}{\left[|y|^{2}+(f(x)-f(x-y))^{2}\right]^{5 / 2}} .
$$

The terms $K_{3}, K_{4}$, and $K_{5}$ are estimated as $J_{1}$. Then we find that

$$
\begin{gathered}
K_{3} \leq C\left(1+\|f\|_{C^{2}}^{2}\right)\|f\|_{H^{4}}^{2}, \\
K_{4} \leq C\left(1+\|f\|_{C^{1}}\|f\|_{C^{2, \delta}}\right)\|f\|_{H^{4}}^{2}
\end{gathered}
$$

and

$$
K_{5} \leq C\left(1+\|f\|_{C^{1}}^{2}\|f\|_{C^{2}}^{2}\right)\|f\|_{H^{4}}^{2}
$$

We rewrite $K_{6}$ and we get

$$
K_{6}=-4 P V \int_{\mathbb{R}^{2}} \partial_{x_{1}}^{4} f(x) S\left(\partial_{x_{2}} \partial_{x_{1}}^{3} f\right)(x) d x
$$


with the operator $S$ defined by

$$
S(g)(x)=P V \int_{|y|<1} \frac{\Sigma(x, y)}{|y|^{2}} g(x-y) d y,
$$

and

$$
\Sigma(x, y)=\frac{y_{2}}{|y|} \frac{\left(\nabla f(x) \cdot \frac{y}{|y|}\right)\left(\nabla \partial_{x_{1}} f(x) \cdot \frac{y}{|y|}\right)}{\left[1+\left(\nabla f(x) \cdot \frac{y}{|y|}\right)^{2}\right]^{5 / 2}} .
$$

The function $\Sigma(x, y)$ satisfies that

$$
\begin{aligned}
& \text { (i) } \quad \Sigma(x, \lambda y)=\Sigma(x, y), \quad \forall \lambda>0, \\
& \text { (ii) } \quad \Sigma(x,-y)=-\Sigma(x, y), \\
& \text { (iii) } \quad \sup _{x}|\Sigma(x, y)| \leq\left\|\nabla \partial_{x_{1}} f\right\|_{L^{\infty}},
\end{aligned}
$$

and therefore $S$ is a bounded linear map on $L^{p}\left(\mathbb{R}^{2}\right)$ for $1<p<\infty$ and $\|S\|_{p} \leq C\left\|\nabla \partial_{x_{1}} f\right\|_{L^{\infty}}$ (see [21] and references therein for more details). Then $K_{6} \leq C\|f\|_{C^{2}}\left\|\partial_{x_{1}}^{4} f\right\|_{L^{2}}\left\|\partial_{x_{2}} \partial_{x_{1}}^{3} f\right\|_{L^{2}}$. We obtain finally

$$
I_{5} \leq C\left(1+\|f\|_{C^{2, \delta}}^{4}\right)\|f\|_{H^{4}}^{2}
$$

In order to estimate the term $I_{6}$ we take

$$
\begin{aligned}
I_{6} & =6 \int_{0}^{1} d s \int_{\mathbb{R}^{2}} d y \int_{\mathbb{R}^{2}} \partial_{x_{1}}^{4} f(x) y \cdot\left(\nabla^{2} \partial_{x_{1}}^{2} f(x+(s-1) y)\right) \cdot y \partial_{x_{1}}^{2} A(x, y) d x \\
& \leq \int_{0}^{1} d s\left(\int_{|y|<1} d y+\int_{|y|>1} d y\right) \int_{\mathbb{R}^{2}}\left|\partial_{x_{1}}^{4} f(x)\left\|\nabla^{2} \partial_{x_{1}}^{2} f(x+(s-1) y)\right\| \partial_{x_{1}}^{2} A(x, y) \| y\right|^{2} d x \\
& \leq C\left(\int_{|y|<1}|y|^{-2+\delta} d y+\int_{|y|>1}|y|^{-3} d y\right)\left(1+\|f\|_{C^{2, \delta}}^{4}\right)\|f\|_{H^{4}}^{2} .
\end{aligned}
$$

The most singular term of $I_{7}$ is $K_{7}$

$$
K_{7}=-12 \int_{\mathbb{R}^{2}} \int_{\mathbb{R}^{2}} \partial_{x_{1}}^{4} f(x)\left(\nabla \partial_{x_{1}} f(x)-\nabla \partial_{x_{1}} f(x-y)\right) \cdot y G(x, y) d y d x,
$$

where

$$
G(x, y)=\frac{(f(x)-f(x-y))\left(\partial_{x_{1}}^{3} f(x)-\partial_{x_{1}}^{3} f(x-y)\right)}{\left[|y|^{2}+(f(x)-f(x-y))^{2}\right]^{5 / 2}} .
$$

Due to $\left|\nabla \partial_{x_{1}} f(x)-\nabla \partial_{x_{1}} f(x-y)\right| \leq\|f\|_{C^{2, \delta}}|y|^{\delta}$ and writing

$$
\partial_{x_{1}}^{3} f(x)-\partial_{x_{1}}^{3} f(x-y)=\int_{0}^{1} \nabla \partial_{x_{1}}^{3} f(x+(s-1) y) \cdot y d s,
$$

we obtain $K_{7} \leq C\left(\|f\|_{C^{2, \delta}}^{2}+1\right)\|f\|_{H^{4}}^{2}$ and $I_{7} \leq C\left(1+\|f\|_{C^{2, \delta}}^{4}\right)\|f\|_{H^{4}}^{2}$.

The most singular term of $I_{8}$ is $K_{8}$

$$
K_{8}=-12 \int_{\mathbb{R}^{2}} \int_{\mathbb{R}^{2}} \partial_{x_{1}}^{4} f(x)\left(\partial_{x_{1}}^{4} f(x)-\partial_{x_{1}}^{4} f(x-y)\right) H(x, y) d y d x,
$$

where

$$
H(x, y)=\frac{(f(x)-f(x-y))(\nabla f(x)-\nabla f(x-y)) \cdot y}{\left[|y|^{2}+(f(x)-f(x-y))^{2}\right]^{5 / 2}} .
$$


Then

$$
K_{8}=-12 \int_{\mathbb{R}^{2}}\left|\partial_{x_{1}}^{4} f(x)\right|^{2} P V \int_{\mathbb{R}^{2}} \frac{(f(x)-f(x-y))(\nabla f(x)-\nabla f(x-y)) \cdot y}{\left[|y|^{2}+(f(x)-f(x-y))^{2}\right]^{5 / 2}} d y d x,
$$

is controlled as before. We obtain $K_{8} \leq C\left(1+\|f\|_{C^{2, \delta}}^{4}\right)\left\|\partial_{x_{1}}^{4} f\right\|_{L^{2}}^{2}$ and $I_{8} \leq C\left(1+\|f\|_{C^{2, \delta}}^{4}\right)\|f\|_{H^{4}}^{2}$.

Finally, we have

$$
\frac{d}{d t}\left\|\partial_{x_{1}}^{4} f\right\|_{L^{2}}^{2}(t) \leq C\left(1+\|f\|_{C^{2, \delta}}^{4}(t)\right)\|f\|_{H^{4}}^{2}(t)
$$

and using Sobolev inequalities we get

$$
\frac{d}{d t}\left\|\partial_{x_{1}}^{4} f\right\|_{L^{2}}^{2}(t) \leq C\left(\|f\|_{H^{4}}^{6}(t)+1\right) .
$$

In a similar way we obtain

$$
\frac{d}{d t}\left\|\partial_{x_{2}}^{4} f\right\|_{L^{2}}^{2}(t) \leq C\left(\|f\|_{H^{4}}^{6}(t)+1\right)
$$

and since we can define $\|f\|_{H^{4}}^{2}=\|f\|_{L^{2}}^{2}+\left\|\partial_{x_{1}}^{4} f\right\|_{L^{2}}^{2}+\left\|\partial_{x_{2}}^{4} f\right\|_{L^{2}}^{2}$, due to (21), (27) and (28) it follows

$$
\frac{d}{d t}\|f\|_{H^{4}}(t) \leq C\left(\|f\|_{H^{4}}^{5}(t)+1\right) .
$$

Using Gronwall's inequality we get that the quantity $\|f\|_{H^{4}}$ is bounded up to a time $T=T\left(\left\|f_{0}\right\|_{H^{4}}\right)$. Then, applying energy methods the local existence result follows.

Let the functions $f_{1}(x, t), f_{2}(x, t)$ be two solutions of equation (13) with $f_{1}(x, 0)=f_{2}(x, 0)=f_{0}(x)$, and $f=f_{1}-f_{2}$. Then

$$
\frac{d}{d t}\|f\|_{L^{2}}^{2}(t)=I_{9}+I_{10}+I_{11}
$$

with

$$
\begin{gathered}
I_{9}=\int_{\mathbb{R}^{2}} f(x) \nabla f(x) \cdot P V \int_{\mathbb{R}^{2}} y\left[|y|^{2}+\left(f_{1}(x)-f_{1}(x-y)\right)^{2}\right]^{-3 / 2} d y d x, \\
I_{10}=-\int_{\mathbb{R}^{2}} f(x) P V \int_{\mathbb{R}^{2}} \nabla f(y, t) \cdot(x-y)\left[|x-y|^{2}+\left(f_{1}(x)-f_{1}(y)\right)^{2}\right]^{-3 / 2} d y d x
\end{gathered}
$$

and

$$
I_{11}=\int_{\mathbb{R}^{2}} f(x) P V \int_{\mathbb{R}^{2}}\left(\nabla f_{2}(x)-\nabla f_{2}(x-y)\right) \cdot y N(x, y) d y d x,
$$

with

$$
N(x, y)=\left[|y|^{2}+\left(f_{1}(x)-f_{1}(x-y)\right)^{2}\right]^{-3 / 2}-\left[|y|^{2}+\left(f_{2}(x)-f_{2}(x-y)\right)^{2}\right]^{-3 / 2} .
$$

Integrating by part in $I_{9}$ we have $I_{9} \leq C\left(\left\|f_{1}\right\|_{H^{4}}\right)\|f\|_{L^{2}}^{2}$, and $I_{11} \leq C\left(\left\|f_{1}\right\|_{H^{4}},\left\|f_{2}\right\|_{H^{4}}\right)\|f\|_{L^{2}}^{2}$. The term

$$
\begin{aligned}
I_{10}= & -\int_{\mathbb{R}^{2}} f(x) P V \int_{\mathbb{R}^{2}} \nabla_{y}(f(y)-f(x)) \cdot(x-y)\left[|x-y|^{2}+\left(f_{1}(x)-f_{1}(y)\right)^{2}\right]^{-3 / 2} d y d x \\
= & -P V \int_{\mathbb{R}^{2}} \int_{\mathbb{R}^{2}} f(x)(f(x)-f(y))\left[|x-y|^{2}+\left(f_{1}(x)-f_{1}(y)\right)^{2}\right]^{-3 / 2} d y d x \\
& +P V \int_{\mathbb{R}^{2}} \int_{\mathbb{R}^{2}} f(x)(f(x)-f(y)) \frac{3\left(f_{1}(x)-f_{1}(y)\right)\left(f_{1}(x)-f_{1}(y)-\nabla f_{1}(x)(x-y)\right)}{\left[|x-y|^{2}+\left(f_{1}(x)-f_{1}(y)\right)^{2}\right]^{5 / 2}} d y d x .
\end{aligned}
$$

Then we have that $I_{10} \leq J_{5}+J_{6}$ where

$$
J_{5}=\int_{\mathbb{R}^{2}}|f(x)|^{2} P V \int_{\mathbb{R}^{2}} \frac{3\left(f_{1}(x)-f_{1}(y)\right)\left(f_{1}(x)-f_{1}(y)-\nabla f_{1}(x)(x-y)\right)}{\left[|x-y|^{2}+\left(f_{1}(x)-f_{1}(y)\right)^{2}\right]^{5 / 2}} d y d x
$$


and

$$
J_{6}=-\int_{\mathbb{R}^{2}} f(x) P V \int_{\mathbb{R}^{2}} f(x-y) \frac{3\left(f_{1}(x)-f_{1}(x-y)\right)\left(f_{1}(x)-f_{1}(x-y)-\nabla f_{1}(x) \cdot y\right)}{\left[|y|^{2}+\left(f_{1}(x)-f_{1}(x-y)\right)^{2}\right]^{5 / 2}} d y d x .
$$

The term $J_{5}$ is estimated as $J_{1}$ obtaining $J_{5} \leq C\left(\left\|f_{1}\right\|_{H^{4}}\right)\|f\|_{L^{2}}^{2}$. The term $J_{6}$ can be expressed as $J_{6}=K_{9}+K_{10}$ with

$$
K_{9}=-3 \int_{\mathbb{R}^{2}} f(x) \int_{\mathbb{R}^{2}} f(x-y) \frac{\left(f_{1}(x)-f_{1}(x-y)\right) G(x, y)}{\left[|y|^{2}+\left(f_{1}(x)-f_{1}(x-y)\right)^{2}\right]^{5 / 2}} d y d x,
$$

where the function $G(x, y)$ is given by

$$
G(x, y)=f_{1}(x)-f_{1}(x-y)-\nabla f_{1}(x) \cdot y-\frac{1}{4} y \cdot\left(\nabla^{2} f_{1}(x)+\nabla^{2} f_{1}(x-y)\right) \cdot y .
$$

One finds that the principal value $K_{10}$ is null. Therefore, we obtain finally $J_{6} \leq C\left(\left\|f_{1}\right\|_{H^{4}}\right)\|f\|_{L^{2}}^{2}$.

Applying Gronwall's inequality we get uniqueness.

\subsection{Case $\Omega=\mathbb{T}^{2}$}

In the periodic case we give the theorem of local well-posedness and the differences with $\Omega=\mathbb{R}^{2}$.

Theorem 4.2 Let $f_{0}(x) \in H^{k}\left(\mathbb{T}^{2}\right)$ for $k \geq 4$ and $\rho_{2}>\rho_{1}$. Then there exists a time $T>0$ so that there is a unique solution to (15) in $C^{1}\left([0, T] ; H^{k}\left(\mathbb{T}^{2}\right)\right)$ with $f(x, 0)=f_{0}(x)$.

The proof is similar to the theorem 4.1 but we must use the properties of the function $L$ in (14). We consider without loss of generality $\rho_{2}-\rho_{1}=4 \pi$. In order to control the evolution of the quantity $\left\|\partial_{x_{1}}^{4} f\right\|_{L^{2}}$, the most singular term is

$$
\begin{aligned}
I= & \int_{\mathbb{T}^{2}} \partial_{x_{1}}^{4} f(x) \int_{\mathbb{T}^{2}} \frac{\left(\nabla \partial_{x_{1}}^{4} f(x)-\nabla \partial_{x_{1}}^{4} f(x-y)\right) \cdot y}{\left[|y|^{2}+(f(x)-f(x-y))^{2}\right]^{3 / 2}} L(y, f(x)-f(x-y)) d y d x \\
= & \int_{\mathbb{T}^{2}} \partial_{x_{1}}^{4} f(x) \nabla \partial_{x_{1}}^{4} f(x) \cdot P V \int_{\mathbb{T}^{2}} y \frac{L(y, f(x)-f(x-y))}{\left[|y|^{2}+(f(x)-f(x-y))^{2}\right]^{3 / 2}} d y d x \\
& -\int_{\mathbb{T}^{2}} \partial_{x_{1}}^{4} f(x) P V \int_{\mathbb{T}^{2}} \frac{\nabla \partial_{x_{1}}^{4} f(y) \cdot(x-y)}{\left[|x-y|^{2}+(f(x)-f(y))^{2}\right]^{3 / 2}} L(x-y, f(x)-f(y)) d y d x \\
= & J_{1}+J_{2} .
\end{aligned}
$$

Integrating by parts

$$
\begin{aligned}
J_{1}= & \frac{3}{2} \int_{\mathbb{T}^{2}}\left|\partial_{x_{1}}^{4} f(x)\right|^{2} P V \int_{\mathbb{T}^{2}} A(x, y) L(y, f(x)-f(x-y)) d y d x \\
& -\frac{1}{2} \int_{\mathbb{T}^{2}}\left|\partial_{x_{1}}^{4} f(x)\right|^{2} P V \int_{\mathbb{T}^{2}} \frac{L_{x_{3}}(y, f(x)-f(x-y))(\nabla f(x)-\nabla f(x-y)) \cdot y}{\left[|y|^{2}+(f(x)-f(x-y))^{2}\right]^{3 / 2}} d y d x \\
= & K_{1}+K_{2},
\end{aligned}
$$

where

$$
A(x, y)=\frac{(f(x)-f(x-y))(\nabla f(x)-\nabla f(x-y)) \cdot y}{\left[|y|^{2}+\left((f(x)-f(x-y))^{2}\right]^{5 / 2}\right.}
$$

and

$$
L_{x_{3}}\left(x_{1}, x_{2}, x_{3}\right)=\partial_{x_{3}} L\left(x_{1}, x_{2}, x_{3}\right) .
$$


Due to $\left|L\left(x_{1}, x_{2}, x_{3}\right)-1\right| \leq C\left|\left(x_{1}, x_{2}, x_{3}\right)\right|$ we have

$$
\begin{aligned}
K_{1} & \leq C\left(1+\|f\|_{C^{2, \delta}}^{4}\right)\|f\|_{H^{4}}^{2}+C\left\|\partial_{x_{1}}^{4} f\right\|_{L^{2}}^{2} \max _{x}\left|P V \int_{\mathbb{T}^{2}} \frac{(\nabla f(x) \cdot y)\left(y \cdot \nabla^{2} f(x) \cdot y\right)}{\left.\left[|y|^{2}+(\nabla f(x) \cdot y)\right)^{2}\right]^{5 / 2}} d y\right| \\
& \leq C\left(1+\|f\|_{C^{2, \delta}}^{4}\right)\|f\|_{H^{4}}^{2} .
\end{aligned}
$$

Using that $|f(x)-f(x-y)| \leq\|f\|_{C^{1}}|y|$ and $L_{x_{3}}=0$ in $\left\{x_{1}^{2}+x_{2}^{2}+x_{3}^{2} \leq 4\right\}$, we have that

$$
\begin{aligned}
K_{2} & =-\frac{1}{2} \int_{\mathbb{T}^{2}}\left|\partial_{x_{1}}^{4} f(x)\right|^{2} \int_{|y|>\frac{2}{1+\|f\|_{C^{1}}}} \frac{L_{x_{3}}(y, f(x)-f(x-y))(\nabla f(x)-\nabla f(x-y)) \cdot y}{\left[|y|^{2}+(f(x)-f(x-y))^{2}\right]^{3 / 2}} d y d x \\
& \leq C\left(\|f\|_{C^{2, \delta}}^{4}+1\right)\|f\|_{H^{4}}^{2} .
\end{aligned}
$$

In order to estimates $J_{2}$, we integrate by parts getting

$$
\begin{aligned}
J_{2} & =-\int_{\mathbb{T}^{2}} \partial_{x_{1}}^{4} f(x) P V \int_{\mathbb{T}^{2}} \frac{\nabla_{y}\left(\partial_{x_{1}}^{4} f(y)-\partial_{x_{1}}^{4} f(x)\right) \cdot(x-y)}{\left[|x-y|^{2}+(f(x)-f(y))^{2}\right]^{3 / 2}} L(x-y, f(x)-f(y)) d y d x \\
& =K_{3}+K_{4}+K_{5}+K_{6}
\end{aligned}
$$

with

$$
\begin{gathered}
K_{3}=-\int_{\mathbb{T}^{2}} \partial_{x_{1}}^{4} f(x) P V \int_{\mathbb{T}^{2}} \frac{\partial_{x_{1}}^{4} f(x)-\partial_{x_{1}}^{4} f(y)}{\left[|x-y|^{2}+(f(x)-f(y))^{2}\right]^{3 / 2}} L(x-y, f(x)-f(y)) d y d x, \\
K_{4}=\int_{\mathbb{T}^{2}} \partial_{x_{1}}^{4} f(x) \int_{\mathbb{T}^{2}}\left(\partial_{x_{1}}^{4} f(x)-\partial_{x_{1}}^{4} f(y)\right) B(x, y) L(x-y, f(x)-f(y)) d y d x, \\
K_{5}=\int_{\mathbb{T}^{2}} \int_{\mathbb{T}^{2}} \partial_{x_{1}}^{4} f(x)\left(\partial_{x_{1}}^{4} f(y)-\partial_{x_{1}}^{4} f(x)\right) C(x, y) L_{x_{3}}(x-y, f(x)-f(y)) d y d x, \\
K_{6}=\int_{\mathbb{T}^{2}} \int_{\mathbb{T}^{2}} \partial_{x_{1}}^{4} f(x)\left(\partial_{x_{1}}^{4} f(y)-\partial_{x_{1}}^{4} f(x)\right) D(x, y) d y d x
\end{gathered}
$$

and

$$
\begin{gathered}
B(x, y)=\frac{3(f(x)-f(y))(f(x)-f(y)-\nabla f(y) \cdot(x-y))}{\left[|x-y|^{2}+(f(x)-f(y))^{2}\right]^{5 / 2}}, \\
C(x, y)=-\frac{\nabla f(y) \cdot(x-y)}{\left[|x-y|^{2}+(f(x)-f(y))^{2}\right]^{3 / 2}}, \\
D(x, y)=-\frac{L_{x_{1}}(x-y, f(x)-f(y))\left(x_{1}-y_{1}\right)+L_{x_{2}}(x-y, f(x)-f(y))\left(x_{2}-y_{2}\right)}{\left[|x-y|^{2}+(f(x)-f(y))^{2}\right]^{3 / 2}}, \\
L_{x_{1}}\left(x_{1}, x_{2}, x_{3}\right)=\partial_{x_{1}} L\left(x_{1}, x_{2}, x_{3}\right), \quad L_{x_{2}}\left(x_{1}, x_{2}, x_{3}\right)=\partial_{x_{2}} L\left(x_{1}, x_{2}, x_{3}\right) .
\end{gathered}
$$

Exchanging the variables $x$ and $y$ we can obtain $K_{3} \leq 0$. The terms $K_{4}, K_{5}$ and $K_{6}$ can be estimated in a similar way as $K_{1}$.

Therefore, we obtain

and analogously

$$
\frac{d}{d t}\left\|\partial_{x_{1}}^{4} f\right\|_{L^{2}}(t) \leq C\left(\|f\|_{H^{4}}^{5}(t)+1\right)
$$

This proves local existence.

$$
\frac{d}{d t}\left\|\partial_{x_{2}}^{4} f\right\|_{L^{2}}(t) \leq C\left(\|f\|_{H^{4}}^{5}(t)+1\right)
$$

The proof of the uniqueness is similar to the case $\Omega=\mathbb{R}^{2}$. 


\section{$4.3 \quad 2-\mathrm{D}$ case}

Using the equation (16) in $\mathbb{R}$ and the equation (17) in the periodic case we obtain the following theorem

Theorem 4.3 Let $f_{0}(x) \in H^{k}$ for $k \geq 3$ and $\rho_{2}>\rho_{1}$. Then there exists a time $T>0$ so that there is a unique solution to (16) in $C^{1}\left([0, T] ; H^{k}\right)$ with $f(x, 0)=f_{0}(x)$.

The proof is similar to the theorem's 4.1 and 4.2 .

\section{Global solution for the 2-D stable case}

In this section we obtain a family of global solutions for the 2-D stable case with a small initial data with respect to a fixed norm. Indeed, we can get the result with an initial data with the property $\left\|f_{0}\right\|_{H^{s}}=\infty$ for $s>3 / 2$.

In this section we consider $x \in \mathbb{R}$ and

$$
\|f\|_{a}=\sum|\hat{f}(k)| e^{a|k|} .
$$

For $a>0$, if $\|f\|_{a}<\infty$, then the function $f$ can be extended analytically on the strip $|\Im z|<a$. Furthermore

$$
\left\|\partial_{x} f\right\|_{a} \leq C \frac{\|f\|_{b}}{b-a}
$$

for $b>a$. The main result of this section is

Theorem 5.1 Let $f_{0}(x)$ be a function such that $\int_{\mathbb{T}} f_{0}(x) d x=0,\left\|\partial_{x} f_{0}\right\|_{0} \leq \varepsilon$ for $\varepsilon$ small enough and

$$
\left\|\partial_{x}^{2} f_{0}\right\|_{b(t)} \leq \varepsilon e^{b(t)}\left(1+|b(t)|^{\gamma-1}\right)
$$

with $0<\gamma<1, b(t)=a-\left(\rho_{2}-\rho_{1}\right) t / 2, \rho_{2}>\rho_{1}$ and $a \leq\left(\rho_{2}-\rho_{1}\right) t / 2$. Then, there exists a unique solution of (16) with $f(x, 0)=f_{0}(x)$ and $\rho_{2}>\rho_{1}$ satisfying

$$
\left\|\partial_{x} f\right\|_{a}(t) \leq C(\varepsilon) \exp \left(\left(2 \sigma a-\left(\rho_{2}-\rho_{1}\right) t\right) / 4\right),
$$

and

$$
\left\|\partial_{x}^{2} f\right\|_{a}(t) \leq C(\varepsilon)\left(1+\left|\sigma a-\frac{\rho_{2}-\rho_{1}}{2} t\right|^{\gamma-1}\right) \exp \left(\left(2 \sigma a-\left(\rho_{2}-\rho_{1}\right) t\right) / 4\right),
$$

for $a \leq \frac{\rho_{2}-\rho_{1}}{2 \sigma} t, \sigma=1+\delta$ and $0<\delta<1$.

The condition (31) can be satisfied for example if $\left\|\Lambda^{1+\gamma} f_{0}\right\|_{0}<\varepsilon$ and $\hat{f}_{0}(0)=\hat{f}_{0}(1)=\hat{f}_{0}(-1)=0$ since

$$
\left\|\partial_{x}^{2} f_{0}\right\|_{b(t)} \leq e^{b(t)}\left\|\Lambda^{1+\gamma} f_{0}\right\|_{0} \max _{k \geq 2}|k|^{1-\gamma} e^{b(t)(|k|-1)} .
$$

In order to prove the theorem, we use the Cauchy-Kowalewski method (see [15] and [16]) in a similar way as Caflisch and Orellana [7] and Siegel, Caflisch and Howison [18]. We show the proof with $\rho_{2}-\rho_{1}=2$ without loss of generality.

Let $g(x, t)$ and $h(x, t)$ be functions satisfying

$$
\begin{aligned}
& g_{t}=-\Lambda g, \\
& g(x, 0)=f_{0}(x), \\
& h_{t}=-\Lambda h+T(g+h), \\
& h(x, 0)=0,
\end{aligned}
$$


with

$$
T(f)=-\pi^{-1} \int_{\mathbb{R}} \frac{\partial_{x} f(x)-\partial_{x} f(x-\alpha)}{\alpha} \frac{\left(\frac{f(x)-f(x-\alpha)}{\alpha}\right)^{2}}{1+\left(\frac{f(x)-f(x-\alpha)}{\alpha}\right)^{2}} d \alpha .
$$

Then the function $f(x, t)=g(x, t)+h(x, t)$ is a solution of $(16)$.

First, we show some properties of the nonlinear operator $T$.

Lemma 5.2 If $\left\|\partial_{x} f\right\|_{a},\left\|\partial_{x} g\right\|_{a}<1$ for $a \geq 0$ then

$$
\begin{gathered}
\widehat{T(f)}(0)=0, \\
\left\|\partial_{x} T(f)\right\|_{a} \leq C_{1}\left\|\partial_{x}^{2} f\right\|_{a}\left\|\partial_{x} f\right\|_{a},
\end{gathered}
$$

and

$$
\begin{aligned}
\left\|\partial_{x} T(f)-\partial_{x} T(g)\right\|_{a} \leq & C_{2}\left(\left\|\partial_{x}^{2} f\right\|_{a}+\left\|\partial_{x}^{2} g\right\|_{a}\right)\left\|\partial_{x} f-\partial_{x} g\right\|_{a} \\
& +C_{2}\left(\left\|\partial_{x} f\right\|_{a}+\left\|\partial_{x} g\right\|_{a}\right)\left\|\partial_{x}^{2} f-\partial_{x}^{2} g\right\|_{a}
\end{aligned}
$$

with $C_{1}=4\left(1-\left\|\partial_{x} f\right\|_{a}^{2}\right)^{-2}$ and $C_{2}=4\left(1-\left\|\partial_{x} f\right\|_{a}^{2}\right)^{-2}+\left(1-\left\|\partial_{x} g\right\|_{a}^{2}\right)^{-2}$.

Proof of the Lemma: Due to the inequality $\left|\partial_{x} f(x)\right| \leq\left\|\partial_{x} f\right\|_{a}<1$ and by (35) we obtain

$$
T(f)=\pi^{-1} \sum_{n \geq 1}(-1)^{n} \int_{\mathbb{R}} \frac{\partial_{x} f(x)-\partial_{x} f(x-\alpha)}{\alpha}\left(\frac{f(x)-f(x-\alpha)}{\alpha}\right)^{2 n} d \alpha,
$$

and

$$
T(f)=\pi^{-1} \partial_{x} \sum_{n \geq 1} \frac{(-1)^{n}}{2 n+1} \int_{\mathbb{R}}\left(\frac{f(x)-f(x-\alpha)}{\alpha}\right)^{2 n+1} d \alpha .
$$

Thus $\widehat{T(f)}(0)=0$. Using $(39)$

$$
\begin{aligned}
\widehat{T(f)}(k) & =\pi^{-1} \sum_{n \geq 1}(-1)^{n} \int_{\mathbb{R}_{k_{0}}, \ldots, k_{2 n}} \delta\left(\sum_{j=0}^{2 n} k_{j}, k\right) i k_{0} \prod_{j=0}^{2 n} \hat{f}\left(k_{j}\right) \frac{1-e^{-i \alpha k_{j}}}{\alpha} d \alpha \\
& =\sum_{n \geq 1}(-1)^{n} \sum_{k_{0}, \ldots, k_{2 n}} \delta\left(\sum_{j=0}^{2 n} k_{j}, k\right) M_{n}\left(k_{0}, \ldots, k_{2 n}\right) i k_{0} \prod_{j=0}^{2 n} \hat{f}\left(k_{j}\right),
\end{aligned}
$$

where

$$
M_{n}\left(k_{0}, \ldots, k_{2 n}\right)=\pi^{-1} \int_{\mathbb{R}} \prod_{j=0}^{2 n} \frac{1-e^{-i \alpha k_{j}}}{\alpha} d \alpha
$$

We get

$$
M_{n}\left(k_{0}, \ldots, k_{2 n}\right)=(-1)^{n} m_{n}\left(k_{0}, \ldots, k_{2 n}\right) \prod_{j=1}^{2 n} k_{j}
$$


with

$$
\begin{aligned}
m_{n}\left(k_{0}, \ldots, k_{2 n}\right)= & \pi^{-1} \int_{0}^{1} d s_{1} \ldots \int_{0}^{1} d s_{2 n} \int_{\mathbb{R}} \frac{1-e^{-i \alpha k_{0}}}{\alpha} \exp \left(i \alpha \sum_{j=1}^{2 n}\left(s_{j}-1\right) k_{j}\right) d \alpha \\
= & \pi^{-1} \int_{0}^{1} d s_{1} \ldots \int_{0}^{1} d s_{2 n} P V \int_{\mathbb{R}} \exp \left(i \alpha \sum_{j=1}^{2 n}\left(s_{j}-1\right) k_{j}\right) \frac{d \alpha}{\alpha} \\
& -\pi^{-1} \int_{0}^{1} d s_{1} \ldots \int_{0}^{1} d s_{2 n} P V \int_{\mathbb{R}} \exp \left(-i \alpha k_{0}+i \alpha \sum_{j=1}^{2 n}\left(s_{j}-1\right) k_{j}\right) \frac{d \alpha}{\alpha} \\
= & i \int_{0}^{1} d s_{1} \ldots \int_{0}^{1} d s_{2 n}(\operatorname{sing} A-\operatorname{sing} B),
\end{aligned}
$$

and

$$
A=\sum_{j=1}^{2 n}\left(s_{j}-1\right) k_{j}, \quad B=-k+\sum_{j=1}^{2 n} s_{j} k_{j} .
$$

It follows

$$
\widehat{T(f)}(k)=\sum_{n \geq 1} \sum_{k_{0}, \ldots, k_{n}} \delta\left(\sum_{j=0}^{2 n} k_{j}, k\right) m_{n}\left(k_{0}, \ldots, k_{2 n}\right) \prod_{j=0}^{2 n} k_{j} \hat{f}\left(k_{j}\right),
$$

with $\left|m_{n}\left(k_{0}, \ldots, k_{2 n}\right)\right| \leq 2$.

We have

$$
\begin{aligned}
\sum_{k} e^{a|k|}|k||\widehat{T(f)}(k)| & \leq 2 \sum_{k} \sum_{n \geq 1} \sum_{k_{0}, \ldots, k_{n}} e^{a|k|}|k| \delta\left(\sum_{j=0}^{2 n} k_{j}, k\right) \prod_{j=0}^{2 n}\left|k_{j}\right|\left|\hat{f}\left(k_{j}\right)\right| \\
& \leq 2 \sum_{n \geq 1}(2 n+1) \sum_{k_{0}, \ldots, k_{n}} e^{a\left|k_{0}\right|}\left|k_{0}\right|^{2}\left|\hat{f}\left(k_{0}\right)\right| \prod_{j=1}^{2 n} e^{a\left|k_{j}\right|}\left|k_{j}\right|\left|\hat{f}\left(k_{j}\right)\right|,
\end{aligned}
$$

and therefore

$$
\left\|\partial_{x} T(f)\right\|_{a} \leq 2\left\|\partial_{x}^{2} f\right\|_{a} \sum_{n \geq 1}(2 n+1)\left\|\partial_{x} f\right\|_{a}^{2 n}=2\left\|\partial_{x}^{2} f\right\|_{a} \frac{3\left\|\partial_{x} f\right\|_{a}^{3}-\left\|\partial_{x} f\right\|_{a}^{4}}{\left(1-\left\|\partial_{x} f\right\|_{a}^{2}\right)^{2}}
$$

We get (37) for $\left\|\partial_{x} f\right\|_{a}<1$. In a similar way we obtain (38)

From (34) $g$ can be expressed as follows

$$
\hat{g}(k, t)=e^{-|k| t} \hat{f}_{0}(k),
$$

and by the hypothesis of the initial data we have

$$
\begin{aligned}
\left\|\partial_{x} g\right\|_{a}(t) & \leq \varepsilon e^{a-t}, \\
\left\|\partial_{x}^{2} g\right\|_{a}(t) & \leq \varepsilon e^{a-t}\left(1+(t-a)^{\gamma-1}\right),
\end{aligned}
$$

for $t \geq a$. We will prove the existence of $h$ by an induction argument on the iterative equation:

$$
\begin{aligned}
& \partial_{t} h^{n+1}=-\Lambda h^{n+1}+T\left(g+h^{n}\right), \\
& h^{n+1}(x, 0)=0, \\
& h^{0}=0,
\end{aligned}
$$


or

$$
\begin{aligned}
& \widehat{h^{n+1}}(k, t)=\int_{0}^{t} e^{-|k|(t-s)}\left(T\left(g+h^{n}\right) \widehat{)}(k, s) d s,\right. \\
& h^{0}=0 .
\end{aligned}
$$

For $h^{1}$ we obtain the following estimates

$$
\left\|\partial_{x} h^{1}\right\|_{a}(t) \leq \int_{0}^{t}\|T(g)\|_{a+s-t}(s) d s=\int_{0}^{t-a}+\int_{t-a}^{t}=I_{1}+I_{2} .
$$

Using (37), (41) and (42) we get

$$
\begin{aligned}
I_{1} & \leq e^{a-t} \int_{0}^{t-a} e^{s}\left\|\partial_{x} T(g)\right\|_{0}(s) d s \leq C e^{a-t} \int_{0}^{t-a} e^{s}\left\|\partial_{x}^{2} g\right\|_{0}(s)\left\|\partial_{x} g\right\|_{0}(s) d s \\
& \leq C \varepsilon^{2} e^{a-t} \int_{0}^{t-a} e^{-s}\left(1+s^{\gamma-1}\right) d s \leq \frac{C \varepsilon^{2}(1+2 \gamma)}{\gamma} e^{a-t} .
\end{aligned}
$$

By (41) and (42) we have

$$
I_{2} \leq C \int_{t-a}^{t}\left\|\partial_{x}^{2} g\right\|_{a+s-t}(s)\left\|\partial_{x} g\right\|_{a+s-t}(s) d s \leq C \varepsilon^{2} e^{2(a-t)} a\left(1+(t-a)^{\gamma-1}\right) \leq \frac{2 C \varepsilon^{2}}{\delta} e^{a-t},
$$

due to the inequalities $(a \delta)^{\gamma-1}>(t-a)^{\gamma-1}$ and $a e^{a-t} \leq \delta^{-1}$ for $\sigma a<t$. Then

$$
\left\|\partial_{x} h^{1}\right\|_{a}(t) \leq \frac{5 C \varepsilon^{2}}{\delta \gamma} e^{a-t}
$$

Choosing $b=a+s-t+\frac{t-a}{2}$ we have

$$
\begin{aligned}
\left\|\partial_{x}^{2} h^{1}\right\|_{a}(t) & \leq \int_{0}^{t}\left\|\partial_{x}^{2} T(g)\right\|_{a+s-t}(s) d s \leq \int_{0}^{t} \frac{\left\|\partial_{x} T(g)\right\|_{b}(s)}{b-(a+s-t)} d s \leq 2 \int_{0}^{t} \frac{\left\|\partial_{x} T(g)\right\|_{b}}{t-a} \\
& \leq\left(\int_{0}^{\frac{t-a}{2}}+\int_{\frac{t-a}{2}}^{t}\right)=I_{3}+I_{4},
\end{aligned}
$$

where

$$
\begin{aligned}
I_{3} & \leq \frac{2 C e^{\frac{a-t}{2}}}{t-a} \int_{0}^{\frac{t-a}{2}} e^{s}\left\|\partial_{x}^{2} g\right\|_{0}(s)\left\|\partial_{x} g\right\|_{0}(s) d s \leq \frac{2 C \varepsilon^{2} e^{\frac{a-t}{2}}}{t-a} \int_{0}^{\frac{t-a}{2}} e^{-s}\left(1+s^{\gamma-1}\right) d s \\
& \leq \frac{2 C \varepsilon^{2}}{\gamma} e^{\frac{a-t}{2}}\left(1+(t-a)^{\gamma-1}\right)
\end{aligned}
$$

and

$$
\begin{aligned}
I_{4} & \leq \frac{2 C}{t-a} \int_{\frac{t-a}{2}}^{t}\left\|\partial_{x}^{2} g\right\|_{b}(s)\left\|\partial_{x} g\right\|_{b}(s) d s \leq \frac{2 C \varepsilon^{2}}{t-a} e^{a-t}\left(1+\left(\frac{t-a}{2}\right)^{\gamma-1}\right)\left(\frac{t}{2}+\frac{a}{2}\right) \\
& \leq \frac{3 C \varepsilon^{2}}{\delta} e^{a-t}\left(1+(t-a)^{\gamma-1}\right) .
\end{aligned}
$$

Therefore 


$$
\begin{aligned}
\left\|\partial_{x} h^{1}\right\|_{a}(t) & \leq \frac{5 C \varepsilon^{2}}{\delta \gamma} e^{a-t}, \\
\left\|\partial_{x}^{2} h^{1}\right\|_{a}(t) & \leq \frac{5 C \varepsilon^{2}}{\delta \gamma} e^{\frac{a-t}{2}}\left(1+(t-a)^{\gamma-1}\right) .
\end{aligned}
$$

Define $r^{n+1}=h^{n+1}-h^{n}$,

$$
R_{n}=\sup _{\substack{0 \leq a<\infty \\ \sigma a<t}}\left(\left\|\partial_{x} r^{n}\right\|_{a}+\frac{\left\|\partial_{x}^{2} r^{n}\right\|_{a}}{1+(t-\sigma a)^{\gamma-1}}\right) e^{\frac{t-\sigma a}{2}}
$$

and

$$
M_{n}=\sup _{\substack{0 \leq a<\infty \\ \sigma a<t}}\left(\left\|\partial_{x} h^{n}\right\|_{a}+\frac{\left\|\partial_{x}^{2} h^{n}\right\|_{a}}{1+(t-\sigma a)^{\gamma-1}}\right) e^{\frac{t-\sigma a}{2}} .
$$

Take $M_{1}=R_{1} \leq \frac{5 C \varepsilon^{2}}{\delta \gamma} \leq \frac{\varepsilon_{0}}{2}$ and suppose that $M_{j}, R_{j} \leq \frac{\varepsilon_{0}}{2}$ for any $j=2, \ldots, n$, then

$$
\left\|\partial_{x} r^{n+1}\right\|_{a} \leq \int_{0}^{t}\left\|\partial_{x} T\left(g+h^{n}\right)-\partial_{x} T\left(g+h^{n-1}\right)\right\|_{a+s-t}(s) d s=\int_{0}^{t-a}+\int_{t-a}^{t}=I_{7}+I_{8} .
$$

Using (38) we have

$$
\begin{aligned}
I_{7} \leq & C e^{a-t} \int_{0}^{t-a} e^{s}\left(\left\|\partial_{x} r^{n}\right\|_{0}(s)\left(\left\|\partial_{x}^{2} g+\partial_{x}^{2} h^{n}\right\|_{0}(s)+\left\|\partial_{x}^{2} g+\partial_{x}^{2} h^{n-1}\right\|_{0}(s)\right)\right. \\
& +C e^{a-t} \int_{0}^{t-a} e^{s}\left(\left\|\partial_{x}^{2} r^{n}\right\|_{0}(s)\left(\left\|\partial_{x} g+\partial_{x} h^{n}\right\|_{0}(s)+\left\|\partial_{x} g+\partial_{x} h^{n-1}\right\|_{0}(s)\right) d s\right. \\
\leq & 2 C \varepsilon_{0} R_{n} e^{a-t} \int_{0}^{t-a}\left(1+s^{\gamma-1}\right) d s \leq \frac{2 C \varepsilon_{0}}{\gamma} R_{n} e^{\frac{\sigma a-t}{2}},
\end{aligned}
$$

and

$$
\begin{aligned}
I_{8} \leq & C \int_{t-a}^{t}\left(\left\|\partial_{x} r^{n}\right\|_{a+s-t}(s)\left(\left\|\partial_{x}^{2} g+\partial_{x}^{2} h^{n}\right\|_{a+s-t}(s)+\left\|\partial_{x}^{2} g+\partial_{x}^{2} h^{n-1}\right\|_{a+s-t}(s)\right) d s\right. \\
& +C \int_{t-a}^{t}\left(\left\|\partial_{x}^{2} r^{n}\right\|_{a+s-t}(s)\left(\left\|\partial_{x} g+\partial_{x} h^{n}\right\|_{a+s-t}(s)+\left\|\partial_{x} g+\partial_{x} h^{n-1}\right\|_{a+s-t}(s)\right) d s\right. \\
\leq & 2 C \varepsilon_{0} R_{n} \int_{t-a}^{t} e^{\delta s-\sigma(t-a)}\left(1+(\sigma(t-a)-\delta s)^{\gamma-1}\right) d s \\
\leq & \frac{2 C \varepsilon_{0}}{\delta} R_{n} \int_{t-\sigma a}^{t-a} e^{-x}\left(1+x^{\gamma-1}\right) d x \leq \frac{6 C \varepsilon_{0}}{\gamma \delta} R_{n} e^{\sigma a-t} .
\end{aligned}
$$

We obtain for $b=a+s-t+\frac{\sigma(t-a)-\delta s}{2 \sigma}$

$$
\begin{aligned}
\left\|\partial_{x}^{2} r^{n+1}\right\|_{a}(t) & \leq \int_{0}^{t}\left\|\partial_{x}^{2}\left(T\left(g+h^{n}\right)-T\left(g+h^{n-1}\right)\right)\right\|_{a+s-t}(s) d s \\
& \leq \int_{0}^{t} \frac{\| \partial_{x}\left(T\left(g+h^{n}\right)-T\left(g+h^{n-1}\right) \|_{b}(s)\right.}{b-(a+s-t)} d s \\
& \leq 2 \sigma \int_{0}^{t} \frac{\| \partial_{x}\left(T\left(g+h^{n}\right)-T\left(g+h^{n-1}\right) \|_{b}\right.}{\sigma(t-a)-\delta s} \leq\left(\int_{0}^{\frac{\sigma}{\sigma+1}(t-a)}+\int_{\frac{\sigma}{\sigma+1}(t-a)}^{t}\right)=I_{9}+I_{10} .
\end{aligned}
$$


We have $\sigma(t-a)-\delta s>\frac{2 \sigma}{\sigma+1}(t-a)$ for $0 \leq s \leq \frac{\sigma}{\sigma+1}(t-a)$ and therefore we obtain

$$
\begin{aligned}
I_{9} \leq & \frac{(\sigma+1) C}{t-a} \int_{0}^{\frac{\sigma}{\sigma+1}(t-a)} e^{b}\left(\left\|\partial_{x} r^{n}\right\|_{0}(s)\left(\left\|\partial_{x}^{2} g+\partial_{x}^{2} h^{n}\right\|_{0}(s)+\left\|\partial_{x}^{2} g+\partial_{x}^{2} h^{n-1}\right\|_{0}(s)\right)\right. \\
& +\frac{(\sigma+1) C}{t-a} \int_{0}^{\frac{\sigma}{\sigma+1}(t-a)} e^{b}\left(\left\|\partial_{x}^{2} r^{n}\right\|_{0}(s)\left(\left\|\partial_{x} g+\partial_{x} h^{n}\right\|_{0}(s)+\left\|\partial_{x} g+\partial_{x} h^{n-1}\right\|_{0}(s)\right) d s\right. \\
\leq & \frac{2(\sigma+1) C \varepsilon_{0}}{t-a} R_{n} \int_{0}^{\frac{\sigma}{\sigma+1}(t-a)} e^{b-s}\left(1+s^{\gamma-1}\right) d s \leq \frac{4 \sigma C \varepsilon_{0}}{\gamma} R_{n} e^{\frac{a-t}{2}}\left(1+(t-a)^{\gamma-1}\right) .
\end{aligned}
$$

Using (38) and the induction hypothesis we get

$$
\begin{aligned}
I_{10} & \leq 4 \sigma C \varepsilon_{0} R_{n} \int_{\frac{\sigma}{\sigma+1}(t-a)}^{t} e^{\sigma b-s} \frac{\left(1+(s-\sigma b)^{\gamma-1}\right)}{\sigma(t-a)-\delta s} d s \\
& \leq 4 \sigma C \varepsilon_{0} R_{n} \int_{\frac{\sigma}{\sigma+1}(t-a)}^{t} e^{\frac{\delta s-\sigma(t-a)}{2}} \frac{1+\left(\frac{\sigma(t-a)-\delta s}{2}\right)^{\gamma-1}}{\sigma(t-a)-\delta s} d s \\
& \leq \frac{4 \sigma C \varepsilon_{0}}{\delta} R_{n} \int_{\frac{t-\sigma a}{2}}^{\frac{\sigma}{\sigma+1}(t-a)} e^{-x}\left(x^{-1}+x^{\gamma-2}\right) d x \leq \frac{8 \sigma C \varepsilon_{0}}{\delta(1-\gamma)} R_{n} e^{\frac{\sigma a-t}{2}}\left(1+(\sigma a-t)^{\gamma-1}\right) .
\end{aligned}
$$

Due to the estimates for $I_{7}, I_{8}, I_{9}$ and $I_{10}$ we obtain

$$
R_{n+1} \leq \frac{C \sigma \varepsilon_{0}}{\delta \gamma(\gamma-1)} R_{n}
$$

Choosing $\varepsilon_{0}$ small enough we get

$$
R_{n+1} \leq \frac{1}{2} R_{n} \leq \ldots \leq \frac{1}{2^{n}} R_{1} \leq \frac{\varepsilon_{0}}{2^{n+1}}
$$

and

$$
M_{n+1} \leq \sum_{j=1}^{n+1} R_{n+1} \leq \varepsilon_{0}
$$

Therefore, we obtain the function $h=\lim _{n \rightarrow \infty} h^{n}$ satisfying

$$
\left\|\partial_{x} h\right\|_{a}(t) \leq \sum_{n} R_{n} e^{\frac{\sigma a-t}{2}} \leq \varepsilon_{0} e^{\frac{\sigma a-t}{2}} .
$$

Taking $f(x, t)=g(x, t)+h(x, t)$, we get $(32)$ for $\rho_{2}-\rho_{1}=2$.

In order to show the uniqueness, we write the equation (13) for $\rho_{2}-\rho_{1}=2$ in the following form

$$
\begin{aligned}
& f_{t}=-\Lambda f+T(f), \\
& f(x, 0)=f_{0}(x) .
\end{aligned}
$$

Suppose that there exist two solutions $f^{1}$ and $f^{2}$ with $f^{1}(x, 0)=f^{2}(x, 0)$. Define $R$ by

$$
R=\sup _{\substack{0 \leq a<\infty \\ \sigma a<t}}\left(\left\|\partial_{x} f^{1}-\partial_{x} f^{2}\right\|_{a}+\frac{\left\|\partial_{x}^{2} f^{1}-\partial_{x}^{2} f^{2}\right\|_{a}}{1+(t-\sigma a)^{\gamma-1}}\right) e^{\frac{t-\sigma a}{2}} .
$$

It follows that $R \leq \frac{C(\varepsilon) \sigma}{\delta \gamma(\gamma-1)} R$ and for $\varepsilon$ small enough it yields $\frac{C(\varepsilon) \sigma}{\delta \gamma(\gamma-1)}<1$ and therefore $f_{1}=f_{2}$. 


\section{Ill-posedness for the unstable case}

Here we show ill-posedness for the unstable case $\rho_{1}>\rho_{2}$. We use the global solution for the 2-D stable case $f\left(x_{1}, t\right)$ satisfying (32) with $\left\|\Lambda^{1+\gamma} f_{0}\right\|_{0}<C$ and $\left\|\Lambda^{1+\gamma+\zeta} f_{0}\right\|_{0}=\infty$ for $\gamma, \zeta>0$. Making a change of variables, we define $f_{\lambda}\left(x_{1}, t\right)=\lambda^{-1} f\left(\lambda x_{1},-\lambda t+\lambda^{1 / 2}\right)$ obtaining $\left\{f_{\lambda}\right\}_{\lambda>0}$ a family of solutions to the unstable case. Using (32) follows

$$
\left\|f_{\lambda}\right\|_{H^{s}}(0)=|\lambda|^{s-\frac{3}{2}}\|f\|_{H^{s}}\left(\lambda^{1 / 2}\right) \leq C|\lambda|^{s-\frac{3}{2}}\|f\|_{1}\left(\lambda^{1 / 2}\right) \leq C|\lambda|^{s-\frac{3}{2}} e^{-\frac{\left|\rho_{2}-\rho_{1}\right|}{4} \lambda^{1 / 2}},
$$

and

$$
\left\|f_{\lambda}\right\|_{H^{s}}\left(\lambda^{-1 / 2}\right)=|\lambda|^{s-\frac{3}{2}}\|f\|_{H^{s}}(0) \geq|\lambda|^{s-\frac{3}{2}} C \sum_{k}|k|^{1+\gamma+\zeta}\left|\hat{f}_{0}(k)\right|=\infty,
$$

for $s>3 / 2$ and $\gamma, \zeta$ small enough. We obtain an ill posed problem for $s>3 / 2$.

Theorem 6.1 Let $s>3 / 2$, then for any $\varepsilon>0$ there exists a solution $f$ of (16) with $\rho_{1}>\rho_{2}$ and $0<\delta<\varepsilon$ such that $\|f\|_{H^{s}}(0) \leq \varepsilon$ and $\|f\|_{H^{s}}(\delta)=\infty$.

Remark 6.2 If one considers a solution of the 3-D problem satisfying $f\left(x_{1}, x_{2}, t\right)=f\left(x_{1}, t\right)$, from the equation (13) one obtains a solution of (16). This shows that solutions of the 2-D case are solutions of the 3-D problem and therefore, using the above theorem, one obtains ill-posedness for the 3-D case with $\rho_{1}>\rho_{2}$.

\section{References}

[1] D. Ambrose. Well-posedness of Two-phase Hele-Shaw Flow without Surface Tension. Euro. Jnl. of Applied Mathematics 15 597-607, 2004.

[2] D. Ambrose and N. Masmoudi. The zero surface tension limit of two-dimensional water waves. Comm. Pure Appl. Math. 58 1287-1315, 2005.

[3] J. Bear, Dynamics of Fluids in Porous Media, American Elsevier, New York, 1972.

[4] A. L. Bertozzi and P. Constantin. Global regularity for vortex patches. Comm. Math. Phys. 152 (1): 19-28, 1993.

[5] A. L. Bertozzi and A. J. Majda. Vorticity and the Mathematical Theory of Incompresible Fluid Flow. Cambridge Press, 2002.

[6] G. Birkhoff. Helmholtz and Taylor instability. Hydrodynamics Instability, Proc. Symp. Appl. Math. XII A.M.S., 55-76, 1962.

[7] R. Caflisch and O. Orellana. Singular solutions and ill-posedness for the evolution of vortex sheets. SIAM J. Math. Anal. 20 (2): 293-307, 1989.

[8] J.Y. Chemin. Persistence of geometric structures in two-dimensional incompressible fluids. Ann. Sci. Ecole. Norm. Sup. 26 (4): 517-542, 1993.

[9] P. Constantin, T.F. Dupont, R.E. Goldstein, L.P. Kadanoff, M.J. Shelley and S.M. Zhou. Droplet breakup in a model of the Hele-Shaw cell. Physical Review E, 47, 4169-4181, 1993.

[10] P. Constantin, A. J. Majda, and E. Tabak. Formation of strong fronts in the 2-D quasigeostrophic thermal active scalar. Nonlinearity, 7:1495-1533, 1994. 
[11] J. Escher and G. Simonett. Classical solutions for Hele-Shaw models with surface tension. Adv. Differential Equations, 2:619-642, 1997.

[12] Hele-Shaw. Nature 58, 34, 1898.

[13] T.Y. Hou, J.S. Lowengrub and M.J. Shelley. Removing the Stiffness from Interfacial Flows with Surface Tension. J. Comput. Phys., 114: 312-338, 1994.

[14] M. Muskat. The flow of homogeneous fluids through porous media. New York, 1937.

[15] L. Nirenberg. An abstract form of the nonlinear Cauchy-Kowalewski theorem. J. Differential Geometry, 6 561-576, 1972.

[16] T. Nishida. A note on a theorem of Nirenberg. J. Differential Geometry, 12 629-633, 1977.

[17] P.G. Saffman and Taylor. The penetration of a fluid into a porous medium or Hele-Shaw cell containing a more viscous liquid. Proc. R. Soc. London, Ser. A 245, 312-329, 1958.

[18] M. Siegel, R. Caflisch and S. Howison. Global Existence, Singular Solutions, and Ill-Posedness for the Muskat Problem. Comm. Pure and Appl. Math., 57: 1374-1411, 2004.

[19] J.L. Rodrigo. On the Evolution of Sharp Fronts for the Quasi-Geostrophic Equation. Comm. Pure and Appl. Math., 58: 0821-0866, 2005.

[20] E. Stein and G. Weiss. Introduction to Fourier Analysis on Euclidean spaces. Princeton University Press. Princeton, NJ, 1971.

[21] E. Stein. Harmonic Analysis. Princeton University Press. Princeton, NJ, 1993.

[22] G. Taylor. The instability of liquid surfaces when accelerated in a direction perpendicular to their planes. I. Proc. Roy. Soc. London. Ser. A. 201, 192-196 1950.

[23] S. Wu. Well-posedness in Sobolev spaces of the full water wave problem in 2-D. Invent. math. 130, 39-72 1997.

[24] S. Wu. Well-posedness in Sobolev spaces of the full water wave problem in 3-D. J. Amer. Math. Soc. 12, 445-495 1999. 